\title{
Nonlinear energy-maximising optimal control of wave energy systems: A moment-based approach
}

\author{
Nicolás Faedo, Giordano Scarciotti, Member, IEEE, Alessandro Astolfi, Fellow, IEEE and John V. Ringwood, \\ Senior Member, IEEE.
}

\begin{abstract}
Linear dynamics are virtually always assumed when designing optimal controllers for wave energy converters (WECs), motivated by both their simplicity and computational convenience. Nevertheless, unlike traditional tracking control applications, the assumptions under which the linearisation of WEC models is performed are challenged by the energy-maximising controller itself, which intrinsically enhances device motion to maximise power extraction from incoming ocean waves. In this paper, we present a moment-based energy-maximising control strategy for WECs subject to nonlinear dynamics. We develop a framework under which the objective function (and system variables) can be mapped to a finite-dimensional tractable nonlinear program, which can be efficiently solved using state-of-the-art nonlinear programming solvers. Moreover, we show that the objective function belongs to a class of generalised convex functions when mapped to the moment-domain, guaranteeing the existence of a global energy-maximising solution, and giving explicit conditions for when a local solution is, effectively, a global maximiser. The performance of the strategy is demonstrated through a case study, where we consider (state and input-constrained) energymaximisation for a state-of-the-art CorPower-like WEC, subject to different hydrodynamic nonlinearities.
\end{abstract}

Index Terms-Wave energy, energy-maximisation, moment, moment-matching, nonlinear optimal control.

\section{INTRODUCTION.}

$\mathbf{E}$ NERGY-MAXIMISING control of wave energy converters (WECs) has been shown to be one of the fundamental contributions towards efficient energy extraction from ocean waves [1], [2]. In particular, optimal control strategies significantly improve maximum time-averaged power extraction from waves, potentially reducing the levelised cost of energy, and hence contributing to the roadmap towards successful commercialisation of WECs.

Any energy-maximising control strategy for WECs must take into account the inherent physical limitations of both the

This work was supported by the Science Foundation Ireland under Grant No. SFI/13/IA/1886 and the Royal Society International Exchange Cost Share programme (IEC $\backslash R 1 \backslash 180018$ ). This work has been partially supported by the European Union's Horizon 2020 Research and Innovation Programme under grant agreement No. 739551 (KIOS CoE).

Nicolás Faedo is with the Centre for Ocean Energy Research, Maynooth University, Co. Kildare, Ireland (corresponding author, e-mail: nicolas.faedo@mu.ie).

John. V. Ringwood is with the Centre for Ocean Energy Research, Maynooth University, Co. Kildare, Ireland (e-mail: john.ringwood@mu.ie).

Giordano Scarciotti is with the Department of Electrical and Electronic Engineering, Imperial College London, London SW7 2AZ, U.K. (e-mail: g.scarciotti@ic.ac.uk).

Alessandro Astolfi is with the Department of Electrical and Electronic Engineering, Imperial College London, London, SW7 2AZ, U.K., and also with the Department of Civil Engineering and Computer Science Engineering, University of Rome Tor Vergata, 00133 Rome, Italy (e-mail: a.astolfi@ic.ac.uk). device itself and the power take-off (PTO) actuator dynamics, such that energy extraction is maximised, while also mimising the risk of component damage. The above control specifications form the basis for a range of studies, which are mostly formulated in terms of an optimal control problem (OCP) subject to both state and input constraints. A comprehensive review of these strategies can be found in [2], [3].

Linear dynamics are virtually always considered when designing optimal controllers for WECs (see [3, Table 3]), motivated by both their simplicity (in terms of formulation and solution of the corresponding $\mathrm{OCP}$ ), and their associated computational convenience. In other words, these model-based control strategies must be computed in real-time ${ }^{1}$, therefore limiting the computational complexity of the hydrodynamic models employed. Moreover, there is also a limit to the complexity of mathematical models for which an optimal control solution can be effectively found, either algebraically or numerically. Another strongly contributing factor to the use of linear dynamics is that linear hydrodynamic theory is a well-established field where considerable effort and refinement has been expended in the calculation of linear hydrodynamic parameters. There is therefore little appetite to extend these models to include nonlinear effects.

Nevertheless, despite the list of motives described above, the linearity assumption has been recently an object of debate (see, for instance, [6], [7]): WECs are, by their nature, prone to show significant and diverse nonlinear effects, since their principal aim, pursued by the optimal control strategy, is to enhance the amplitude of motion to maximise power extraction. In other words, and in contrast to traditional set-point tracking control systems, where the control system ensures that the system operation is around the setpoint and actively attempts to reduce the variance around this point, the assumptions under which the linearisation of WEC models is performed are challenged by the controller itself, particularly in relation to small movements around the equilibrium position. This may, in certain conditions, return poor results, both in terms of accuracy of motion prediction that in terms of power production assessment [6], which are the key variables involved in any energy-maximising OCP.

This provides significant motivation for optimal control strategies that can effectively handle nonlinear effects, both in terms of the well-posedness of the OCP (i.e. existence of global energy-maximising solutions), and real-time capabili-

\footnotetext{
${ }^{1}$ Though beyond the scope of this study, we note that control techniques which optimise control parameters/laws offline have been also proposed within the WEC literature. The reader is referred to, for instance, [4], [5].
} 
ties. Some attempts to address this problem have already been offered within the WEC control literature. We provide a brief review in the following paragraphs.

The papers [8] and [9] propose one of the first nonlinear optimal control formulations in the WEC literature: nonlinear energy-maximising model predictive control (MPC) applied to (different) heaving point absorbers. The nonlinear effects considered in [8] arise from the forces exerted by the mooring system utilised to attach the device to the seafloor. Nevertheless, nonlinear hydrodynamic effects, such as viscous forces (which are significant in this type of device [7]) are disregarded, whilst (non-parametric) radiation forces are completely neglected. Concerning computational efficiency, the authors specifically claim that the study in [8] does not focus on real-time applicability, but rather on highlighting the advantages of using nonlinear MPC compared to its linear counterpart. A follow up of this study can be found in [10], where the discretisation for the MPC is modified (from a zero-order- to a second-order- hold), and a state estimator is incorporated. On a different note, while the authors of [9] assume linear dynamics for the WEC, they compute the control force using a nonlinear MPC formulation given by a time-varying system (written in explicit form), which naturally induces a time-varying objective function.

The papers [11], [12] and [13] apply pseudospectral optimal control methods (see, for instance, [14] for a review) to different WEC technologies, subject to a number of nonlinearities: viscous forces ( [11] and [12]), nonlinear hydrostatic restoring force ( [13] and [12]), and a non-ideal PTO system ( [11]). Although these pseudospectral formulations are indeed appealing from a computational perspective, the results in [11], [12] and [13] do not guarantee existence of solutions to the proposed OCPs. Moreover, even if a solution is found, it is not clear under which conditions this energy-maximising control law is effectively a globally optimal solution.

Recently, a novel energy-maximising optimal control framework for WECs was presented in [15], [16]. This approach is based on the concept of moment (discussed in Section II) and maps the original energy-maximising optimal control problem into a concave quadratic program (QP), systematically guaranteeing a unique solution for the original energy-maximising control objective, subject to both state and input constraints. Nevertheless, the mathematical framework in [15], [16] considers WECs subject to linear dynamics, hence hindering the application of this strategy to more realistic scenarios in which nonlinear effects become significant, as previously discussed in this section.

Exploiting the concept of nonlinear moment introduced in [17], this paper presents an energy-maximising control strategy for WECs subject to nonlinear dynamics. In particular, we propose an approximation method which allows us to efficiently parameterise the energy-maximising OCP subject to both state and input constraints. To this end the paper provides the following contributions:

- We propose a method to map the objective function (and system variables) to a finite-dimensional tractable nonlinear program (NP), which can be efficiently solved using state-of-the-art nonlinear programming solvers (see, for instance, [18]).

- By showing that the objective function arising from the proposed moment-based strategy belongs to a family of approximately convex/concave mappings (particularly to the so-called outer $\Gamma$-convex/concave [19] functions), we guarantee the existence of a global energy-maximising solution, under mild assumptions.

- In analogy to the case of convex/concave functions, where each local solution is also global, we give explicit conditions to determine whether a local energy-maximising solution is, effectively, a global maximiser for the proposed moment-based OCP, having strong practical implications when numerically solving the associated NP.

- Finally, we present an extensive case study based on the energy-maximisation problem for a state-of-the-art CorPower-like WEC subject to hydrodynamic nonlinearities.

The remainder of this paper is organised as follows. Section II recalls key theoretical concepts behind the nonlinear moment-based framework. Section III describes and formalises the energy-maximising problem for WECs subject to nonlinear dynamics, while Section IV details the main contributions of this paper, i.e. the proposed nonlinear moment-based optimal control strategy. Finally, Section V discusses the application of this approach to a state-of-the-art CorPower-like heaving WEC, while Section VI encompasses the main conclusions of this study.

\section{A. Notation and Preliminaries.}

Standard notation is considered throughout this study. $\mathbb{R}^{+}$ $\left(\mathbb{R}^{-}\right)$denotes the set of non-negative (non-positive) real numbers. $\mathbb{C}^{0}$ denotes the set of pure-imaginary complex numbers. The symbol 0 stands for any zero element, dimensioned according to the context. The notation $\mathbb{N}_{q}$ indicates the set of all positive natural numbers up to $q$, i.e. $\mathbb{N}_{q}=\{1,2, \ldots, q\}$. The symbol $\mathbb{I}_{n}$ denotes the identity matrix of order $n$, while the notation $\mathbf{1}_{n \times m}$ is used to denote a $n \times m$ Hadamard identity matrix (i.e. a $n \times m$ matrix with all its entries equal to 1$)$. The spectrum of a matrix $A \in \mathbb{R}^{n \times n}$, i.e. the set of its eigenvalues, is denoted by $\lambda(A)$. The superscript $\mathrm{T}$ denotes the transposition operator. The symbol $\bigoplus$ denotes the direct sum of $n$ matrices, i.e. $\bigoplus_{i=1}^{n} A_{i}=\operatorname{diag}\left(A_{1}, A_{2}, \ldots, A_{n}\right)$. The notation $\Re\{z\}$ and $\Im\{z\}$, with $z \in \mathbb{C}$, stands for the real-part and the imaginary-part operators, respectively. The symmetric-part of a matrix $A \in \mathbb{R}^{n \times n}$ is defined (and denoted) as $\mathscr{H}\{A\}=\left(A+A^{\top}\right) / 2$. The Frobenius norm of a matrix $X \in \mathbb{R}^{n \times m}$ is denoted as $\|X\|_{F}$, while the Euclidean norm of a row vector $V \in \mathbb{R}^{n}$ is denoted as $\|V\|_{2}$. The generalised Dirac- $\delta$ function, shifted by $t_{j} \in \mathbb{R}^{+}$, is denoted as $\delta_{t_{j}}=\delta\left(t-t_{j}\right)$. The Kronecker product between two matrices $M_{1} \in \mathbb{R}^{n \times m}$ and $M_{2} \in \mathbb{R}^{p \times q}$ is denoted by $M_{1} \otimes M_{2} \in \mathbb{R}^{n p \times m q}$. The convolution between two functions $f$ and $g$ over the set $\Omega \subset \mathbb{R}$, i.e. $\int_{\Omega} f(\tau) g(t-\tau) d \tau$ is denoted as $f * g$. Let $f$ and $g$ be two functions belonging to the Hilbert space $L^{2}(\Xi)=\left\{f:\left.\Xi \rightarrow \mathbb{R}\left|\int_{\Xi}\right| f(\tau)\right|^{2} d \tau<+\infty\right\}$, where $\Xi \subset \mathbb{R}$ is closed. Then the inner-product between $f$ 
and $g$ is given by $\langle f, g\rangle=\int_{\Xi} f(\tau) g(\tau) d \tau$. The span of the set $\mathscr{X}=\left\{x_{i}\right\}_{i=1}^{k} \subset \mathscr{Z}$, where $\mathscr{Z}$ is a vector space over the field $\mathbb{R}$, is denoted as $\operatorname{span}\{\mathscr{X}\}$. The closed ball contained in $\mathbb{R}^{n}$, with center $z \in \mathbb{R}^{n}$ and radius $r \in \mathbb{R}^{+}$, is defined as $\mathscr{B}(z, r)=\left\{y \in \mathbb{R}^{n} \mid\|y-z\|_{2} \leq r\right\}$. Finally, the symbol $\varepsilon_{n} \in \mathbb{R}^{n}$ denotes a vector with odd entries equal to 1 and even entries equal to 0 .

In the remainder of this section, the formal definitions of two important operators are presented, since their definition in the literature can often be ambiguous.

Definition 1 (Kronecker sum). [20] The Kronecker sum of two matrices $P_{1}$ and $P_{2}$, with $P_{1} \in \mathbb{R}^{n \times n}$ and $P_{2} \in \mathbb{R}^{k \times k}$, is defined (and denoted) as

$$
P_{1} \hat{\oplus} P_{2} \triangleq P_{1} \otimes \mathbb{I}_{k}+\mathbb{I}_{n} \otimes P_{2}
$$

Definition 2 (Vec operator). [20] Given a matrix $P=$ $\left[p_{1}, p_{2}, \ldots, p_{m}\right] \in \mathbb{R}^{n \times m}$, where $p_{j} \in \mathbb{R}^{n}, j \in \mathbb{N}_{m}$, the vector valued operator vec is defined as

$$
\operatorname{vec}\{P\} \triangleq\left[\begin{array}{c}
p_{1} \\
p_{2} \\
\vdots \\
p_{m}
\end{array}\right] \in \mathbb{R}^{n m}
$$

Finally, we recall a useful property of the vec operator.

Property 1. [20] Let $P_{3} \in \mathbb{R}^{n \times m}$ and $P_{4} \in \mathbb{R}^{m \times q}$. Then

$$
\operatorname{vec}\left\{P_{3} P_{4}\right\}=\left(\mathbb{I}_{q} \otimes P_{3}\right) \operatorname{vec}\left\{P_{4}\right\}=\left(P_{4}^{\top} \otimes \mathbb{I}_{n}\right) \operatorname{vec}\left\{P_{3}\right\} .
$$

\section{MOMENT-BASED THEORY}

To keep this paper reasonably self-contained, this section briefly recalls some of the key concepts behind the momentbased framework, as developed in [17], [21]. In particular (after recalling a set of standing assumptions) this section is mainly concerned with the definition of moment for nonlinear systems.

We consider a nonlinear, single-input single-output, continuous-time, system described, for $t \in \mathbb{R}^{+}$, by the set of equations ${ }^{2}$

$$
\begin{aligned}
& \dot{x}=f(x, u), \\
& y=h(x),
\end{aligned}
$$

with $x(t) \in \mathbb{R}^{n}, u(t) \in \mathbb{R}, y(t) \in \mathbb{R}$, and $f: \mathbb{R}^{n} \times \mathbb{R} \rightarrow \mathbb{R}^{n}$ and $h: \mathbb{R}^{n} \rightarrow \mathbb{R}$ sufficiently smooth mappings defined in the neighbourhood of the origin of $\mathbb{R}^{n}$. Assume that the origin is an equilibrium point of (4), i.e. $f(0,0)=0$ and $h(0)=0$.

Consider now a signal generator (often also referred to as exogenous system [22]) described by the equations

$$
\begin{gathered}
\dot{\xi}=S \xi, \\
u=L \xi,
\end{gathered}
$$

${ }^{2}$ From now on, we drop the dependence on $t$ when clear from the context. with $\xi(t) \in \mathbb{R}^{\nu}, S \in \mathbb{R}^{\nu \times \nu}$ and $L^{\top} \in \mathbb{R}^{\nu}$, and the so-called composite (or interconnected) system

$$
\begin{aligned}
\dot{\xi} & =S \xi, \\
\dot{x} & =f(x, L \xi), \\
y & =h(x) .
\end{aligned}
$$

Following [17], [21], we now introduce a set of standing assumptions to formalise the definition of moment. Recall that a system is minimal if it is observable, accessible and descibed by analytic mappings (see [21, Chapter 2]).

Assumption 1. There exists a mapping $\pi$, locally ${ }^{3}$ defined in a neighborhood of $\xi=0$ and with $\pi(0)=0$, which solves the partial differential equation

$$
\frac{\partial \pi(\xi)}{\partial \xi} S \xi=f(\pi(\xi), L \xi) .
$$

Assumption 2. System (4) is minimal and the pair $(S, L)$ is observable.

Definition 3. [17], [21] Consider system (4) and the signal generator (5). Suppose Assumptions 1 and 2 hold. The mapping $h \circ \pi$ is the moment of system (4) at $(S, L)$.

Finally, we recall a result which connects the definition of moment (as in Definition 3) with the steady-state response of the output of the interconnected system (6) (as in Figure 1), introducing an additional assumption concerning the dynamics of (5).

Assumption 3. The signal generator (5) is such that all eigenvalues of $S$ are simple and with zero real part. Moreover, the pair $(S, \xi(0))$ is excitable ${ }^{4}$.

Remark 1. For linear systems excitability is equivalent to reachability, i.e. with $\xi(0)$ playing the role of the input matrix, see [23].

Theorem 1. [17], [21]. Consider system (4) and the signal generator (5). Suppose Assumptions 2 and 3 hold and that the zero equilibrium of the system (4) is locally exponentially stable. Then Assumption 1 holds, and the moment of system (4), computed along a trajectory $\xi(t)$, coincides with the steadystate response of the output of the interconnected system (6), i.e. $y_{\mathrm{ss}}(t)=h(\pi(\xi(t)))$.

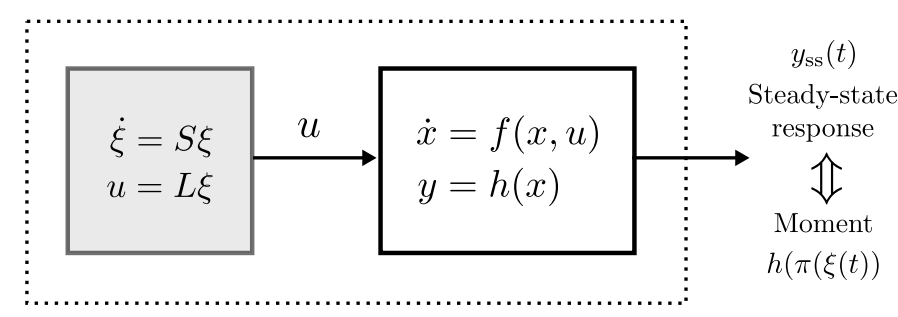

Fig. 1. Block diagram of the composite system (6), showing the relationship between the steady-state output and the moment.

\footnotetext{
${ }^{3}$ Statements are local, though global versions can be directly derived. ${ }^{4}$ We refer the reader to [23] for the definition of excitability.
} 


\section{PRoblem FORMUlation: OPtimal CONTROL FOR WECS.}

WEC optimal control design entails an energy-maximisation criterion, where the objective is to maximise the absorbed energy from ocean waves over a finite time interval $\Xi=[0, T] \subset$ $\mathbb{R}^{+}$. To be precise, the useful energy absorbed from incoming waves is converted in the PTO system, and can be directly computed as the time integral of converted (instantaneous) power, i.e. this energy-maximising control procedure can be cast as an optimal control problem, with objective function $\mathcal{J}$ defined as

$$
\mathcal{J}(u)=\frac{1}{T} \int_{\Xi} u(\tau) \dot{x}(\tau) d \tau,
$$

where $\dot{x}: \Xi \rightarrow \mathbb{R}$ and $u: \Xi \rightarrow \mathbb{R}$ denote the velocity of the device and control (PTO) force, respectively.

We now briefly recall the basics behind control-oriented WEC modelling in Section III-A, to later pose the optimal control problem for wave energy systems subject to the motion (state and input) constraints in Section III-B.

\section{A. WEC dynamics.}

We begin this section by recalling (see e.g. [24]) well-known facts behind control-oriented WEC modelling. For simplicity we assume a 1-degree-of-freedom (DoF) device and we recall that a similar analysis can be carried out for multi-DoF devices following the moment-based multiple-input, multiple-output approach presented in [16].

Remark 2. In what follows, to simplify notation, the terminology associated to a 1-DoF translational device is employed with some minor abuse. This is without loss of generality, and the same analysis can be carried out for a rotational 1-DoF device.

The equation of motion for such a WEC can be expressed in the time-domain, in terms of Newton's second law, as

$$
m \ddot{x}=\mathcal{F}_{r}+\mathcal{F}_{h}^{l}+\mathcal{F}_{e}+\mathcal{F}^{n l}-u,
$$

where $m$ is the mass of the buoy, $x$ the device excursion (displacement), $\mathcal{F}_{e}$ the wave excitation force (external input), $\mathcal{F}_{h}^{l}$ the linear component of the hydrostatic restoring force, $\mathcal{F}_{r}$ the radiation force, and $u$ the exerted control (PTO) force. The mapping $\mathcal{F}^{n l}$ represent potential sources of nonlinearity depending on $x$ and $\dot{x}$, such as viscous drag force and nonlinear hydrostatic effects (see Section V-A). Note that, in line with the assumptions in Section II, it is assumed that the mapping $\mathcal{F}^{n l}$ is sufficiently smooth ${ }^{5}$. The linear component of the hydrostatic force can be written as $\mathcal{F}_{h}^{l}(t)=-s_{h} x(t)$, where $s_{h}=\rho g_{r} D$ denotes the hydrostatic stiffness, with $\rho$ the water density, $D$ the characteristic area of the device, and $g_{r}$ the gravitational constant. The radiation force $\mathcal{F}_{r}$ is modelled based on linear potential theory and, using the well-known Cummins' equation [26], can be written as

$$
\mathcal{F}_{r}(t)=-\mu_{\infty} \ddot{x}(t)-\int_{\mathbb{R}^{+}} k(\tau) \dot{x}(t-\tau) d \tau,
$$

\footnotetext{
${ }^{5}$ If the mapping $\mathcal{F}^{n l}$ is non-smooth, smooth approximations can be used to apply the framework proposed in our study. Note that smooth approximations have been previously exploited, within the WEC control literature, for example in [25].
}

where $\mu_{\infty}=\lim _{\omega \rightarrow+\infty} \tilde{A}(\omega)>0$ is the added-mass at infinite frequency, $\tilde{A}(\omega)$ is the radiation added mass ${ }^{6}$ and $k: \mathbb{R}^{+} \rightarrow \mathbb{R}^{+}$is the (causal) radiation impulse response function containing the memory effect of the fluid response. Finally, the equation of motion of the WEC is given by

$$
\mathcal{M} \ddot{x}+k * \dot{x}+s_{h} x-\mathcal{F}^{n l}=\mathcal{F}_{e}-u,
$$

with $\mathcal{M}=m+\mu_{\infty}$. We note that equation (11) is of a Volterra integro-differential form, specifically of the convolution class ${ }^{7}$.

\section{B. Physical limitations: state and input constraints.}

As it is well-known in the wave energy literature, the unconstrained energy-maximising optimal control law, i.e. the maximiser of $\mathcal{J}$ in (8), often requires unrealistic device motion and excessively high PTO (control) forces, which consign this optimal unconstrained solution to the academic realm, far from being practically viable. Aiming to derive an implementable solution, we consider constraints on both the displacement and the velocity of the WEC, $x$ and $\dot{x}$, and on the exerted control force $u$. This guarantees that the physical limits associated with device and actuator dynamics are consistently respected, while effectively maximising, at the same time, absorbed energy from incoming waves. This set of constraints can be compactly written as

$$
\left\{\begin{array}{l}
|x(t)| \leq X_{\max } \\
|\dot{x}(t)| \leq V_{\max } \\
|u(t)| \leq U_{\max }
\end{array}\right.
$$

with $t \in \mathbb{R}^{+}$, and where $\left\{X_{\max }, V_{\max }, U_{\max }\right\} \subset \mathbb{R}^{+}$. Having knowledge of the control objective function defined in (8) (i.e. the mapping $\mathcal{J}$ ), the nonlinear dynamics of the WEC in (11), and the set of state and input constraints defined in (12), the constrained energy-maximising OCP can be posed as follows.

Problem 1 (Energy-maximising OCP). Find an optimal control input $u^{\mathrm{opt}}: \Xi \rightarrow \mathbb{R}$ such that

$$
\begin{aligned}
& u^{\text {opt }}=\arg \max _{u} \mathcal{J}, \\
& \text { subject to: } \\
& \left\{\begin{array}{l}
\text { nonlinear WEC dynamics (11), } \\
\text { state and input constraints (12). }
\end{array}\right.
\end{aligned}
$$

Remark 3. We assume that the set of state and input constraints in (13) are feasible, i.e. (12) is given in terms of a feasible set (region). We refer the reader to, for instance, [28], for an explicit analysis and discussion of the feasibility problem for WEC energy-maximising control systems, as a function of the wave excitation input.

\section{Nonlinear Moment-Based WEC Control.}

In this section we present the main contributions of the paper. First, we develop a method to find approximate solutions to the problem of determining the moment of nonlinear

\footnotetext{
${ }^{6}$ See [24] for the definition of $\tilde{A}(\omega)$.

${ }^{7}$ The interested reader is referred to [27] for further detail on this class of integro-differential operators.
} 
systems describing WECs. Second, we formulate and solve the unconstrained optimal control problem in the moment-domain. Third, we extend the solution to the state and input constrained problem.

\section{A. Approximation of moments for nonlinear systems}

We begin by rewriting the equation of motion (11) in state space as

$$
\begin{aligned}
& \dot{\varphi}=f(\varphi, \mathscr{U})=A \varphi-B(k * C \varphi)+B \mathscr{U}+f_{n l}(\varphi), \\
& y=h(\varphi)=C \varphi,
\end{aligned}
$$

where $\varphi(t)=\left[\varphi_{1}(t), \varphi_{2}(t)\right]^{\top}=[x(t), \dot{x}(t)]^{\top} \in \mathbb{R}^{2}$, is the state-vector of the continuous-time model and $y=\dot{x}$ is the output of the system (assuming the velocity as the measurable output of the device). The function $\mathscr{U}: \mathbb{R}^{+} \rightarrow \mathbb{R}$, defined as the input of system (14), is given by the expression ${ }^{8}$

$$
\mathscr{U}=\mathcal{F}_{e}-u \text {. }
$$

Under this representation, the triple of matrices $(A, B, C)$ and the nonlinear mapping $f_{n l}: \mathbb{R}^{2} \rightarrow \mathbb{R}^{2}$ forming the equation of motion (14) are given by

$$
\begin{aligned}
A & =\left[\begin{array}{cc}
0 & 1 \\
-s_{h} \mathcal{M}^{-1} & 0
\end{array}\right], B=\left[\begin{array}{c}
0 \\
\mathcal{M}^{-1}
\end{array}\right], C^{\top}=\left[\begin{array}{l}
0 \\
1
\end{array}\right], \\
f_{n l}(\varphi) & =\left[\begin{array}{c}
0 \\
\mathcal{F}^{n l}(\varphi)
\end{array}\right] .
\end{aligned}
$$

We recall, from [30], that the standard assumption for the mathematical representation of wave excitation forces in ocean engineering applications is that $\mathcal{F}_{e}$ can be written as a finite sum of harmonics of a so-called fundamental frequency $\omega_{0}$. Following the moment-based theory presented in Section II, the wave excitation force input can be written as a signal generator described, for $t \in \mathbb{R}^{+}$, by the set of equations

$$
\begin{gathered}
\dot{\xi}=S \xi, \\
\mathcal{F}_{e}=L_{e} \xi,
\end{gathered}
$$

where $\xi(t) \in \mathbb{R}^{\nu}, L_{e}^{\top} \in \mathbb{R}^{\nu}$ and the dynamic matrix $S \in \mathbb{R}^{\nu \times \nu}$ can be written in block-diagonal form as

$$
S=\bigoplus_{p=1}^{\tilde{f}}\left[\begin{array}{cc}
0 & p \omega_{0} \\
-p \omega_{0} & 0
\end{array}\right]
$$

with $\nu=2 \tilde{f}, \tilde{f}>0$ integer.

Remark 4. To simplify the notation used throughout the upcoming results, and to explicitly focus this manuscript on the formulation of a nonlinear moment-based controller, it is assumed that the moment-domain equivalent $L_{e}$, characterising the wave excitation $\mathcal{F}_{e}$ as in equation (17), is known, i.e. full (instantaneous and future) knowledge of $\mathcal{F}_{e}$ is available over the time interval $\Xi \subset \mathbb{R}^{+}$. This is without loss of generality, since estimation and forecasting algorithms for $\mathcal{F}_{e}$ (which are often required due to the inherent difficulty behind measuring wave excitation forces in a moving body [31]) can

\footnotetext{
${ }^{8}$ Similar arguments can be adopted for multi-DoF systems. The reader is referred to [16], [29] for further detail.
}

be incorporated straightforwardly, by following the adaptation of the moment-based representation of $\mathcal{F}_{e}$ for receding-horizon control presented in [32, Section IV-A], without further modifications.

Even though the wave excitation force is composed of $\tilde{f}$ harmonics multiple of the (angular) fundamental frequency $\omega_{0}$, it is convenient (for the subsequent theoretical results) to assume that the control input $u$ can be composed of a higher number $\tilde{f}+d$ of harmonics, with $d>0$ integer. For this purpose, we define the auxiliary 'extended' signal generator as follows. Let $\bar{S} \in \mathbb{R}^{(\nu+\iota) \times(\nu+\iota)}$ be such that

$$
\bar{S}=S \oplus\left(\bigoplus_{p=1}^{d}\left[\begin{array}{cc}
0 & (p+\tilde{f}) \omega_{0} \\
-(p+\tilde{f}) \omega_{0} & 0
\end{array}\right]\right),
$$

with $\iota=2 d$. We can now express the wave excitation force and the control input $u$ as a function of this extended signal generator as

$$
\begin{aligned}
\dot{\bar{\xi}} & =\bar{S} \bar{\xi}, \\
\mathcal{F}_{e} & =\left[L_{e} 0\right] \bar{\xi}=\bar{L}_{e} \bar{\xi}, \\
u & =\bar{L}_{u} \bar{\xi},
\end{aligned}
$$

where $\bar{\xi}(t) \in \mathbb{R}^{\nu+\iota}$, and $\bar{\xi}(0)=\left[\xi(0)^{\top}, \xi^{\star}(0)^{\top}\right]^{\top}, \xi^{\star}(0) \in \mathbb{R}^{\iota}$. Note that the input defined in (15) can be expressed accordingly as $\mathscr{U}=\left(\bar{L}_{e}-\bar{L}_{u}\right) \bar{\xi}$.

Remark 5. The signal generator (20) is an extension of the one defined in (17) in the sense that it inherently incorporates the matrix $S$, while adding $d$ harmonics multiple of the fundamental frequency $\omega_{0}$. With the selected initial condition $\bar{\xi}(0)$, the wave excitation force $\mathcal{F}_{e}$ can be written as a function of $\bar{\xi}$ by simply using an appropriate inclusion mapping, i.e. completing $L_{e}$ with zeros accordingly.

In preparation for the upcoming results we introduce, without loss of generality, three further assumptions. The first one is on the signal generator defined in equation (20).

Assumption 4. The triple of matrices $\left(\bar{L}_{e}-\bar{L}_{u}, \bar{S}, \bar{\xi}(0)\right)$ is minimal, i.e. observable and excitable.

Note that the previous assumption is without loss of generality as the signal generator is user-defined and so it can always be constructed such that the assumption holds.

Remark 6. Let $\bar{\xi}_{i}$ be the $i$-th entry of $\bar{\xi}$, with $i \in \mathbb{N}_{\nu+\iota}$, and define the set $\overline{\mathscr{X}}=\left\{\bar{\xi}_{i}\right\}_{i=1}^{\nu+\iota}$. Note that, if Assumption 4 holds, then the pair $(\bar{S}, \bar{\xi}(0))$ is excitable and it is straightforward to check that $\operatorname{span}\{\overline{\mathscr{X}}\}=\operatorname{span}\left\{\left\{\cos \left(p \omega_{0} t\right),-\sin \left(p \omega_{0} t\right)\right\}_{p=1}^{\tilde{f}+d}\right\}$. As a consequence, the input $\mathscr{U}$ is always $T$-periodic, where $T=2 \pi / \omega_{0} \in \mathbb{R}^{+}$is the fundamental period of $\mathscr{U}$.

We now state the following standard assumption on the nonlinear mapping $f_{n l}$, to later prove existence and uniqueness of the moment of system (14) at the signal generator $\left(\bar{S}, \bar{L}_{e}-\bar{L}_{u}\right)$.

Assumption 5. The mapping $f_{n l}: \mathbb{R}^{2} \rightarrow \mathbb{R}^{2}$ is such that

$$
f_{n l}(0)=0,\left.\quad \frac{\partial f_{n l}(\varphi)}{\partial \varphi}\right|_{\varphi=0}=0
$$

Note that the assumption is without loss of generality because the matrices in (14) and the mapping $f_{n l}$ can always 
be redefined to satisfy it. Finally, we introduce an assumption on the stability in the first approximation of system (14).

Assumption 6. The zero equilibrium of system

$$
\dot{\varphi}=A \varphi-B(k * C \varphi)
$$

is asymptotically stable.

As discussed in several studies, such as [24], [33], the linear equation of motion (22) is asymptotically stable for any meaningful values of the involved parameters (and impulse response function $k$ ). Thus, this assumption is, in practice, also without loss of generality.

Proposition 1. Suppose Assumptions 4, 5 and 6 hold. Then there exists a mapping $\pi$ which solves the partial differential equation

$$
\frac{\partial \pi(\bar{\xi})}{\partial \bar{\xi}} \bar{S} \bar{\xi}=f\left(\pi(\bar{\xi}),\left(\bar{L}_{e}-\bar{L}_{u}\right) \bar{\xi}\right) .
$$

Moreover, the moment of system (14) at the signal generator $\left(\bar{S}, \bar{L}_{e}-\bar{L}_{u}\right)$ computed along a particular trajectory $\bar{\xi}(t)$ coincides with the well-defined steady-state output response of the interconnected system (14)-(16)-(20), i.e. $y_{\mathrm{ss}}(t)=h(\pi(\bar{\xi}(t)))$.

Proof. Note that, under Assumption 4, the triple of matrices $\left(\bar{L}_{e}-\bar{L}_{u}, \bar{S}, \bar{\xi}(0)\right)$ is minimal. Moreover, the extended signal generator defined in (20) is such that $\lambda(S) \subset \mathbb{C}^{0}$ with simple eigenvalues, in line with Assumption 3. Therefore, Proposition 1 automatically holds as long as the zero equilibrium of the system $\dot{\varphi}=f(\varphi, 0)$ is locally exponentially stable (see Theorem 1). Since this is the case by Assumption 6, the proof is concluded.

In slightly different words, Proposition 1 guarantees that the steady-state response of system (14), driven by (20), can be effectively computed using the corresponding moment at $\left(\bar{S}, \bar{L}_{e}-\bar{L}_{u}\right)$. Nevertheless, even if the existence of $\pi$ (solution of (23)) is guaranteed, it is virtually impossible to compute its analytic expression when the mapping $f$ in (14) is nonlinear.

The very nature of the mapping $\pi$ intrinsically depends on both the characteristics of the signal generator (20) and the system dynamics defined by $f$. Aiming to formally characterise $\pi$, we introduce the following key remarks, which drive the next main result.

Remark 7. Let $\Xi$ be defined as $\Xi=[0, T] \subset \mathbb{R}^{+}$. Note that the set $\overline{\mathscr{X}}$, defined in Remark 6, belongs to the Hilbert space $L^{2}(\Xi)$ and is orthogonal under the standard inner-product operator. Moreover, if Assumption 4 holds, we can always complete $\overline{\mathscr{X}}$ to an orthogonal basis $\mathscr{X}$ of $L^{2}(\Xi)$, i.e. we define (see [34, Chapter 8])

$$
\begin{gathered}
\mathscr{X}=\overline{\mathscr{X}} \cup \breve{\mathscr{X}}, \\
\breve{\mathscr{X}}=\left\{\cos \left(p \omega_{0} t\right),-\sin \left(p \omega_{0} t\right)\right\}_{p=\tilde{f}+d+1}^{\infty}=\left\{\breve{\mathscr{X}}_{i}\right\}_{i=\nu+\iota+1}^{\infty} .
\end{gathered}
$$

Remark 8. If Assumption 4 holds, one can always find a set of mappings $\mathcal{I}_{i}: \mathbb{R}^{\nu+\iota} \rightarrow \mathbb{R}$ such that $\breve{\mathscr{X}}_{i}=\mathcal{I}_{i}(\bar{\xi})$, for every $i>\nu+\iota$ integer. This (standard) result states that we can always generate the elements of the set $\breve{\mathscr{X}}$ (i.e. higher order harmonics of the fundamental frequency $\omega_{0}$ ) by solely operating on the $\nu+\iota$ trigonometric polynomials defined by the entries of $\bar{\xi}$ (see, for example, [35]).

Proposition 2. Suppose Assumptions 4, 5 and 6 hold. Then each element of the mapping $\pi$, as the solution to (23), i.e. $\pi_{k}$, $k \in \mathbb{N}_{2}$, belongs to the Hilbert space $L^{2}(\Xi)$ with $\Xi=[0, T] \subset$ $\mathbb{R}^{+}$, where $T=2 \pi / \omega_{0}$, i.e. it can be uniquely expressed as

$$
\pi_{k}(\bar{\xi})=\sum_{i=1}^{\nu+\iota} \alpha_{k_{i}} \bar{\xi}_{i}+\epsilon_{k}=\bar{\Pi}_{k} \bar{\xi}+\epsilon_{k},
$$

where $\epsilon_{k}=\sum_{i=\nu+\iota+1}^{\infty} \alpha_{k_{i}} \mathcal{I}_{i}(\bar{\xi}), \alpha_{k_{i}} \in \mathbb{R} \forall i$, with $\mathcal{I}_{i}$ as defined in Remark 8 , and the matrix $\bar{\Pi}_{k}^{\top} \in \mathbb{R}^{\nu+\iota}$ is given by $\bar{\Pi}_{k}=\left[\alpha_{k_{1}}, \ldots, \alpha_{k_{\nu+\iota}}\right]$.

Proof. Given the nature of the signal generator defined in equation (20), the function $\mathscr{U}$ is $T$-periodic, with $T=2 \pi / \omega_{0}$ (see Remark 6). Moreover, under the above assumptions, the zero equilibrium of $\dot{\varphi}=f(\varphi, 0)$ is locally exponentially stable and its (well-defined) steady-state solution is also $T$ periodic [36, Section VI], i.e. $\varphi_{\mathrm{ss}}(t)=\varphi_{\mathrm{ss}}(t-T)$. Since under Assumptions 4, 5 and 6, $\varphi_{\mathrm{ss}}(t)=\pi(\bar{\xi}(t))$ (see Proposition $1)$, it is straightforward to conclude that each element of the mapping $\pi$ belongs to $L^{2}(\Xi)$, i.e. it can be expressed as a unique linear combination of the orthogonal basis $\mathscr{X}$ (as defined in Remark 7), which concludes the proof.

Remark 9. The result of Proposition 2 allows $\pi$ to be compactly expressed as

$$
\pi(\bar{\xi})=\left[\begin{array}{l}
\bar{\Pi}_{1} \\
\bar{\Pi}_{2}
\end{array}\right] \bar{\xi}+\left[\begin{array}{l}
\epsilon_{1} \\
\epsilon_{2}
\end{array}\right]=\bar{\Pi} \bar{\xi}+E,
$$

where the term $E: \mathbb{R}^{\nu+\iota} \rightarrow \mathbb{R}^{2}$ is called the truncation error.

Note that, if we 'ignore' the truncation error $E$, the mapping $\pi$ can be effectively approximated as $\bar{\pi}(\bar{\xi})=\bar{\Pi} \bar{\xi}$, i.e. by its expansion on the $(\nu+\iota)$-dimensional set $\overline{\mathscr{X}}$. This motivates the following key definition.

Definition 4. We call the function $C \bar{\pi}$, where $\bar{\pi}(\bar{\xi})=\bar{\Pi} \bar{\xi}$, the approximated moment ${ }^{9}$ of system (14) at the signal generator $\left(\bar{S}, \bar{L}_{e}-\bar{L}_{u}\right)$. In addition, we refer to the matrix $\bar{Y}=C \bar{\Pi}$ as the approximated moment-domain equivalent ${ }^{10}$ of $y$.

Remark 10. Under the same set of assumptions as Proposition 2, the approximated moment-domain equivalent of $y$ can be effectively used to approximate the steady-state output of system (14) driven by $\left(\bar{S}, \bar{L}_{e}-\bar{L}_{u}\right)$, i.e. $y_{\mathrm{ss}}(t) \approx C \bar{\Pi} \bar{\xi}(t)=$ $\bar{Y} \bar{\xi}(t)$.

Aiming to propose a method to compute $\bar{Y}$, and inspired by the family of mean weighted residual methods [38], [39], we define the residual mapping $\mathcal{R}: \mathbb{R}^{2} \rightarrow \mathbb{R}^{2}$ as

$$
\mathcal{R}(\bar{\Pi} \bar{\xi}):=\bar{\Pi} \bar{S} \bar{\xi}-f\left(\bar{\Pi} \bar{\xi},\left(\bar{L}_{e}-\bar{L}_{u}\right) \bar{\xi}\right),
$$

which directly arises from 'replacing' $\pi$ with $\bar{\pi}$ in equation (23). Using this residual equation we consider a collocation approach [39, Chapter 4] to compute the approximated moment-domain equivalent $\bar{Y}=C \bar{\Pi}$. In other words, we force

\footnotetext{
${ }^{9}$ This notion is analogous to the one given in [37].

${ }^{10}$ This definition is analogous to that used in the linear moment-based WEC control studies [15] and [16].
} 
equation (26) to be exactly zero at a finite set of collocation points. We make this approximation method explicit in the following proposition.

Proposition 3. Consider the nonlinear system (14) and the signal generator defined by equation (20). Suppose Assumptions 4, 5 and 6 hold. Then, the approximated moment-domain equivalent of $y$ can be computed as $C \bar{\Pi}$, where $\bar{\Pi}$ is the solution of the algebraic system of equations

$\left(\bar{\Pi} \bar{S}-A \bar{\Pi}+B C \bar{\Pi} \mathscr{R}-B\left(\bar{L}_{e}-\bar{L}_{u}\right)\right)\left\langle\bar{\xi}, \delta_{t_{j}}\right\rangle-\left\langle f_{n l}(\bar{\Pi} \bar{\xi}), \delta_{t_{j}}\right\rangle=0$,

with $\mathcal{T}_{\delta}=\left\{t_{i}\right\}_{i=1}^{\nu+\iota} \subset \Xi$ a set of uniformly-distributed time instants, and where the matrix $\mathscr{R} \in \mathbb{R}^{(\nu+\iota) \times(\nu+\iota)}$ is defined as

$$
\mathscr{R}=\bigoplus_{p=1}^{\tilde{f}+d}\left[\begin{array}{rr}
\Re\left\{K\left(p \omega_{0}\right)\right\} & \Im\left\{K\left(p \omega_{0}\right)\right\} \\
-\Im\left\{K\left(p \omega_{0}\right)\right\} & \Re\left\{K\left(p \omega_{0}\right)\right\}
\end{array}\right],
$$

with $K: \mathbb{R} \rightarrow \mathbb{C}, \omega \mapsto K(\omega)$, the Fourier transform of the impulse response function associated with radiation effects, i.e. $k$ in equation (14).

Proof. Note that, using (14), the residual equation (26) can be equivalently written as

$$
\left(\bar{\Pi} \bar{S}-A \bar{\Pi}-B\left(\bar{L}_{e}-\bar{L}_{u}\right)\right) \bar{\xi}+B(k * C \bar{\Pi} \bar{\xi})-f_{n l}(\bar{\Pi} \bar{\xi}),
$$

where the convolution operation involved, associated with the effect of radiation forces acting on the device, can be shown to be such that [15], [16]

$$
k * C \bar{\Pi} \bar{\xi}=C \bar{\Pi} \mathscr{R} \bar{\xi},
$$

with $\mathscr{R}$ as in equation (28). Then, following the well-known collocation method [39, Chapter 4], the residual function is forced to be orthogonal (under the standard inner-product of $L^{2}(\Xi)$ ) to the set of translated Dirac- $\delta$ functions $\left\{\delta_{t_{i}}\right\}_{i=1}^{\nu+\iota}$. Equation (27) follows after considering the superposition property of the inner-product operator.

Corollary 1. The system of algebraic equations (27) can be equivalently written in matrix form as

$$
\left(\bar{\Pi} \bar{S}-A \bar{\Pi}+B C \bar{\Pi} \mathscr{R}-B\left(\bar{L}_{e}-\bar{L}_{u}\right)\right)-F_{n l}(\bar{\Pi}) \Omega^{-1}=0,
$$

where the matrices $F_{n l}(\bar{\Pi}) \in \mathbb{R}^{2 \times(\nu+\iota)}$ and $\Omega \in \mathbb{R}^{(\nu+\iota) \times(\nu+\iota)}$ are defined as

$$
\begin{aligned}
\Omega & =\left[\begin{array}{lll}
\bar{\xi}\left(t_{1}\right) & \ldots & \bar{\xi}\left(t_{\nu+\iota}\right)
\end{array}\right] \\
F_{n l}(\bar{\Pi}) & =\left[\begin{array}{lll}
f_{n l}\left(\bar{\Pi} \bar{\xi}\left(t_{1}\right)\right) & \ldots & f_{n l}\left(\bar{\Pi} \bar{\xi}\left(t_{\nu+\iota}\right)\right)
\end{array}\right] .
\end{aligned}
$$

Proof. Note that if the set $\left\{t_{j}\right\} \subset \Xi$, then $\left\langle f, \delta_{t_{j}}\right\rangle=f\left(t_{j}\right)$, for any continuous function $f: \Xi \rightarrow \mathbb{R}$. Then, the result follows as a consequence of the excitability of the pair $(\bar{S}, \xi(0))$, which implies that the matrix $\Omega$ is always full rank [23].

Remark 11. If the set of uniformly-distributed time instants $\mathcal{T}_{\delta}$ is chosen such that $t_{k}=-T / 2+T k /(\nu+\iota), t_{k} \in \mathcal{T}_{\delta}$ for all $k \in \mathbb{N}_{\nu+\iota}$, then the collocation approach utilised in Proposition 3 is identical to the Galerkin method [39, Chapter 4]. The main advantage of Proposition 3 (collocation) lies in its simplicity of implementation, i.e. we simply use function evaluation (see Corollary 1).
Remark 12. In the light of Remark 11, standard results of Galerkin methods (see [40]) apply to this WEC case. In particular, the existence of solutions to system (27) (equivalently equation (31)), under the hypothesis of Proposition 3, is always guaranteed for all sufficiently large $\iota$. Moreover, the approximated moment $\bar{\pi}(\bar{\xi})=\bar{\Pi} \bar{\xi}$ converges uniformly towards the exact solution (25) as $\iota \rightarrow \infty$ (see also [40]).

We now present a corollary, which illustrates the result of Proposition 3 (through Corollary 1) in a more convenient form for the upcoming nonlinear moment-based energy-maximising control formulation. In particular, we show that equation (31) can be fully expressed in terms of the approximated momentdomain equivalent $\bar{Y}=C \bar{\Pi}$, effectively reducing the number of variables involved in such an equation.

Corollary 2. The system of algebraic equations (31) can be fully written as a function of the approximated moment-domain equivalent $\bar{Y}=C \bar{\Pi}$ as

$$
\bar{Y}-\left(\bar{L}_{e}-\bar{L}_{u}\right) \bar{\Phi}^{\top}+\operatorname{vec}\left\{F_{n l}(g(\bar{Y}))\right\}^{\top} \Phi_{\Omega}^{\top}=0,
$$

where $\bar{\Phi} \in \mathbb{R}^{(\nu+\iota) \times(\nu+\iota)}$ and $\Phi_{\Omega} \in \mathbb{R}^{(\nu+\iota) \times 2(\nu+\iota)}$ are given by the expressions

$$
\begin{aligned}
\bar{\Phi} & =\left(\mathbb{I}_{\nu+\iota} \otimes C\right) \Phi^{-1}\left(\mathbb{I}_{\nu+\iota} \otimes-B\right), \\
\Phi_{\Omega} & =\left(\mathbb{I}_{\nu+\iota} \otimes C\right) \Phi^{-1}\left(\Omega^{-1^{\top}} \otimes \mathbb{I}_{2}\right), \\
\Phi & =\bar{S} \hat{\oplus} A+\mathscr{R}^{\top} \otimes-B C,
\end{aligned}
$$

and the mapping $g: \mathbb{R}^{1 \times(\nu+\iota)} \rightarrow \mathbb{R}^{2 \times(\nu+\iota)}$ is defined as

$$
g(\bar{Y})=\left(\mathbb{I}_{2} \otimes \bar{Y}\right)\left[\begin{array}{l}
\bar{S}^{-1} \\
\mathbb{I}_{\nu+\iota}
\end{array}\right] .
$$

Proof. A direct application of the vec operator (and Property 1) to equation (32), yields

$$
\begin{aligned}
& \operatorname{vec}\{\bar{\Pi}\}+ \Phi^{-1}\left(\mathbb{I}_{\nu+\iota} \otimes B\right) \operatorname{vec}\left\{\bar{L}_{e}-\bar{L}_{u}\right\}+ \\
& \Phi^{-1}\left(\Omega^{-1^{\top}} \otimes \mathbb{I}_{2}\right) \operatorname{vec}\left\{F_{n l}(\bar{\Pi})\right\}=0
\end{aligned}
$$

in which we make explicit use of the skew-symmetricity of $\bar{S}$ i.e. $-\bar{S}^{\top}=\bar{S}$ to obtain $\Phi$ as in (34). The invertibility of the matrix $\Phi$ has been shown in [15], [16]. Equation (33) follows after multiplying both sides of (36) by $\left(\mathbb{I}_{\nu+\iota} \otimes C\right)$, where we note that $\operatorname{vec}\{C \bar{\Pi}\}=\operatorname{vec}\{\bar{Y}\}=\bar{Y}^{\top}$ and $\operatorname{vec}\left\{\bar{L}_{e}-\bar{L}_{u}\right\}=$ $\left(\bar{L}_{e}-\bar{L}_{u}\right)^{\top}$. Finally, the mapping $g$ arises as a result of [41, Proposition 1]: given that $\dot{\varphi}_{1}=\varphi_{2}=y$ in (14), $\bar{\Pi}$ can be written in terms of $\bar{Y}$ simply as

$$
\bar{\Pi}=\left[\begin{array}{c}
\bar{Y} \bar{S}^{-1} \\
\bar{Y}
\end{array}\right]=\left(\mathbb{I}_{2} \otimes \bar{Y}\right)\left[\begin{array}{l}
\bar{S}^{-1} \\
\mathbb{I}_{\nu+\iota}
\end{array}\right]=g(\bar{Y}),
$$

which concludes our proof.

Remark 13. If $F_{n l}(g(\bar{Y}))=0$, i.e. system (14) is linear, the approach of Proposition 3 (through Corollary 2) recovers (without approximation) the linear moment-domain equivalent $\bar{Y}=\left(\bar{L}_{e}-\bar{L}_{u}\right) \bar{\Phi}^{\top}$, presented in [15], [16]. In other words, equation (33) can be regarded as the linear moment-domain equivalent of $y$ plus a nonlinear 'perturbation' term. 


\section{B. Motion unconstrained nonlinear moment-based OCP}

The results presented in Section IV-A can be effectively used to approximate the energy-maximising optimal control problem presented in Definition 1, making explicit use of the connection between moments and the steady-state behaviour of system (14). In the following, we provide a definition of the so-called approximated energy-maximising $O C P$, using the approximated moment-domain equivalent $\bar{Y}$, presented in Definition 4. Note that we do not yet include the set of state and input constraints defined in (12). These are explicitly incorporated in Section IV-C.

Problem 2 (Approximated energy-maximising OCP). Suppose Assumptions 4, 5 and 6 hold. Find the optimal control input $\bar{u}^{\text {opt }}=\bar{L}_{u}^{\text {opt }} \bar{\xi}$ such that

$$
\begin{aligned}
& \bar{L}_{u}^{\text {opt }}=\arg \max _{\bar{L}_{u}^{\top} \in \mathbb{R}^{\nu+\iota}} \frac{1}{T} \int_{\Xi} \bar{L}_{u} \bar{\xi}(\tau) \bar{Y} \bar{\xi}(\tau) d \tau, \\
& \quad \text { subject to: } \\
& \quad \bar{Y}-\left(\bar{L}_{e}-\bar{L}_{u}\right) \bar{\Phi}^{\top}+\operatorname{vec}\left\{F_{n l}(g(\bar{Y}))\right\}^{\top} \Phi_{\Omega}^{\top}=0,
\end{aligned}
$$

where $\bar{Y}$ is the approximated moment-domain equivalent of the output of system (14) (see Definition 4 ), and $\bar{\xi}$ is the solution of (20).

Remark 14. The main idea behind Problem 2 relies on substituting the integro-differential (equality) constraint, corresponding with the nonlinear WEC dynamics (14), by the algebraic equation (33). Note that the latter characterises the approximated moment-domain equivalent of the velocity of the device $\dot{x}=y$ (which is the key state variable involved in the energy-maximising objective function (8)). In other words, the approximated OCP posed in Problem 2 explicitly utilises an approximation of the steady-state (output) behaviour of system (14), parameterised in terms of $\bar{Y}$, i.e. $\dot{x}_{\mathrm{ss}}=y_{\mathrm{ss}}(t) \approx \bar{Y} \bar{\xi}(t)$ (see Remark 10), to solve for the corresponding optimal control input $\bar{u}^{\text {opt }}$, in terms of the signal generator (20).

Remark 15. Following Remark 12, if $\iota \rightarrow \infty$, then the steady-state output response of system (14) is exactly given by $y_{\mathrm{ss}}(t)=\bar{Y} \bar{\xi}(t)$, and the algebraic equality constraint in the OCP of Problem 2 corresponds to the exact steady-state motion of the device, without approximations.

Based on Problem 2, we are now ready to propose a solution to the motion unconstrained energy-maximising optimal control problem, i.e. (13) without considering input and state constraints (see Problem 2), in terms of a specific tractable finite-dimensional nonlinear program (NP). This claim is formalised in the following proposition.

Proposition 4 (Nonlinear moment-based unconstrained NP). Suppose Assumptions 4, 5 and 6 hold, and let $\bar{\xi}(0)=\varepsilon_{\nu+\iota}$. Then, for $\iota$ sufficiently large, the solution of the (motion unconstrained) approximated energy-maximising OCP, posed in Problem 2, can be computed as $\bar{u}^{\text {opt }}=\bar{L}_{u}^{\text {opt }} \xi$, where

$$
\bar{L}_{u}^{\mathrm{opt}}=-\left(\bar{Y}^{\mathrm{opt}}+\operatorname{vec}\left\{F_{n l}\left(g\left(\bar{Y}^{\mathrm{opt}}\right)\right)\right\}^{\top} \Phi_{\Omega}^{\top}-\bar{L}_{e}\right) \bar{\Phi}^{-1^{\top}},
$$

and the matrix $\bar{Y}^{\mathrm{opt}}$ is the solution of the finite-dimensional nonlinear program

$$
\bar{Y}^{\text {opt }}=\arg \max _{\bar{Y}^{\top} \in \mathbb{R}^{\nu+\iota}} \overline{\mathcal{J}}_{Q P}(\bar{Y})+\overline{\mathcal{J}}_{n l}(\bar{Y}),
$$

with $\overline{\mathcal{J}}_{\mathrm{QP}}: \mathbb{R}^{\nu+\iota} \rightarrow \mathbb{R}, \overline{\mathcal{J}}_{n l}: \mathbb{R}^{\nu+\iota} \rightarrow \mathbb{R}$ defined as

$$
\begin{aligned}
& \overline{\mathcal{J}}_{\mathrm{QP}}(\bar{Y})=-\frac{1}{2} \bar{Y} \bar{\Phi}^{-1} \bar{Y}^{\top}+\frac{1}{2} \bar{Y} \bar{L}_{e}^{\top}, \\
& \overline{\mathcal{J}}_{n l}(\bar{Y})=-\frac{1}{2} \bar{Y} \bar{\Phi}^{-1} \Phi_{\Omega} \operatorname{vec}\left\{F_{n l}(g(\bar{Y}))\right\} .
\end{aligned}
$$

Proof. The fundamental step towards this proof lies in [15, Proposition 3]. In particular, due to the (harmonic) nature of the signal generator defined in equation (20), the objective function corresponding with the approximated OCP, i.e. equation (38), can be equivalently written [15] as

$$
\overline{\mathcal{J}}=\frac{1}{T} \int_{\Xi} \bar{Y} \bar{\xi}(\tau) \bar{L}_{u} \bar{\xi}(\tau) d \tau=\frac{1}{2} \bar{Y} \bar{L}_{u}^{\top},
$$

for $\bar{\xi}(0)=\varepsilon_{\nu+\iota}$. Substituting $\bar{L}_{u}$ in (42), using the result of Corollary 2 , we can write $\overline{\mathcal{J}}$, as a function of $\bar{Y}$, as

$$
\begin{aligned}
& \overline{\mathcal{J}}=-\frac{1}{2} \bar{Y} \bar{\Phi}^{-1} \bar{Y}^{\top}+\frac{1}{2} \bar{Y} \bar{L}_{e}^{\top}-\frac{1}{2} \bar{Y} \bar{\Phi}^{-1} \Phi_{\Omega} \operatorname{vec}\left\{F_{n l}(g(\bar{Y}))\right\} \\
& \overline{\mathcal{J}}=\overline{\mathcal{J}}_{\mathrm{QP}}(\bar{Y})+\overline{\mathcal{J}}_{n l}(\bar{Y})
\end{aligned}
$$

where the optimal control input $\bar{u}^{\text {opt }}=\bar{L}_{u}^{\text {opt }} \bar{\xi}$ can be straightforwardly recovered using the equality (33), yielding equation (39), which concludes our proof.

Proposition 4 explicitly uses the approximated momentdomain equivalent $\bar{Y}$ to propose a finite-dimensional tractable optimisation problem, allowing for the computation of an energy-maximising control solution for the approximated OCP posed in Problem 2, when the WEC is subject to nonlinear dynamics. Note that there is (almost) no restriction on the nature of the mapping $f_{n l}$, so that a general class of nonlinear effects can be considered, including complex hydrodynamic nonlinearities, such as those discussed in Section V.

Remark 16. The moment-based NP stated in Proposition 4 has to be carried out over the approximated moment-domain equivalent $\bar{Y}^{\top} \in \mathbb{R}^{\nu+\iota}$ only, i.e. in terms of the momentdomain representation of the velocity of the device, and can be solved using efficient state-of-the-art numerical routines, such as interior-point methods (IPMs) [42].

Remark 17. There is an intrinsic trade-off between the degree of accuracy behind the approximated OCP, controlled by the parameter $\iota$ (see Remark 15), and the underlying computational complexity of equation (40). In other words, a higher $\iota$ results in improved energy absorption, but also intrinsically increases the computational requirements of the strategy.

Remark 18. If $\overline{\mathcal{J}}_{n l}(\bar{Y})=0$, Proposition 4 recovers the optimal moment-based control input proposed in [15] for the linear WEC case. To be precise, if there are no nonlinearities involved in (14), equation (40) is of a concave quadratic type, i.e. a quadratic program $(\mathrm{QP})$ written as

$$
\bar{Y}_{l}^{\text {opt }}=\arg \max _{\bar{Y}^{\top} \in \mathbb{R}^{\nu+\iota}}-\frac{1}{2} \bar{Y} \bar{\Phi}^{-1} \bar{Y}^{\top}+\frac{1}{2} \bar{Y} \bar{L}_{e}^{\top},
$$

where the function $\overline{\mathcal{J}}_{\mathrm{QP}}$ is strictly concave for any physically meaningful parameters involved in the WEC equation of motion (14) (see [15, Proposition 4]). 
Following Remark 18, it is straightforward to note that the NP stated in Proposition 4 can be seen as a QP problem characterised by the objective function $\overline{\mathcal{J}}_{\mathrm{QP}}$, and 'perturbed' by the action of the nonlinear mapping $\overline{\mathcal{J}}_{n l}$. Nevertheless, unlike the linear moment-based energy-maximising OCP of [15], [16] (recalled herein in Remark 18), there is no guarantee that the nonlinear OCP of Proposition 4 admits a global maximiser. Aiming to secure the existence of a global solution to problem (40), we introduce the following standing assumption, to later formalise an appropriate proposition guaranteeing the existence of a global energy-maximising solution to (40).

Assumption 7. The mapping $\overline{\mathcal{J}}_{n l}: \mathbb{R}^{1 \times(\nu+\iota)} \rightarrow \mathbb{R}$ is bounded by a parameter $\alpha_{n l} \in \mathbb{R}^{+}$, i.e.

$$
\sup _{\bar{Y} \boldsymbol{T} \in \mathbb{R}^{\nu+\iota}}\left|\overline{\mathcal{J}}_{n l}(\bar{Y})\right| \leq \alpha_{n l}<+\infty .
$$

As demonstrated in Section V, Assumption 7 is without loss of generality, since the mapping $\varphi \mapsto \mathcal{F}^{n l}(\varphi)$, which effectively defines the nonlinear nature of $\overline{\mathcal{J}}_{n l}$ (see equation (41)), is sufficiently smooth (see Section III-A), and hence bounded on any compact set.

Proposition 5. Suppose Assumption 7 holds. Then, the NP with objective function $\overline{\mathcal{J}}$ defined in Proposition 4 always admits a global maximum $\bar{Y}^{\mathrm{opt}}$.

Proof. The key concept behind this proposition lies in the decomposition of $\overline{\mathcal{J}}$ as in equation (41), i.e. as the sum of a concave problem $\overline{\mathcal{J}}_{\mathrm{QP}}$ and, under Assumption 7, a bounded perturbation $\overline{\mathcal{J}}_{n l}$. To be precise, if $\overline{\mathcal{J}}_{n l}$ is bounded, then the function $-\overline{\mathcal{J}}$ is strictly outer $\Gamma$-convex (see Definition 5 and Theorem 2 in Appendix A), for $\Gamma \subset \mathbb{R}^{1 \times(\nu+\iota)}$, where the set $\Gamma$ is given by

$$
\Gamma=\mathscr{B}(0, r), \quad r=\sqrt{\frac{2 \alpha_{n l}}{\min \lambda\left(\mathscr{H}\left\{\bar{\Phi}^{-1}\right\}\right)}} .
$$

Finally, given that the mapping $\overline{\mathcal{J}}_{\mathrm{QP}}$ has a unique global maximiser $\bar{Y}_{l}^{\text {opt }}$ (see Remark 18), and the set $\Gamma \cap \mathbb{R}^{1 \times(\nu+\iota)}$ is closed, the NP defined by the objective function $\overline{\mathcal{J}}$ always admits a global optimal solution $\bar{Y}^{\text {opt }}$ (see [43, Lemma 4.3] and Property 3 in Appendix A).

Proposition 5 makes explicit use of the strictly outer $\Gamma$ convexity of the function $-\overline{\mathcal{J}}$ to ensure existence of a global solution to the moment-based energy-maximising OCP proposed in this study. In other words, in contrast to stateof-the-art nonlinear WEC control techniques [9]-[13], we explicitly guarantee existence of globally optimal solutions to the associated energy-maximising OCP.

Remark 19. Informally, the concept of outer $\Gamma$-convexity, introduced in [19], is a formalisation of the concept of convexity for a class of functions which are 'roughly' convex, i.e. they possess properties similar to those of convex functions [43]. The reader is referred to Definition 5 in Appendix A for a formal treatment of this concept.

Moreover, recalling key theoretical results from [43], we can use the following property of strictly outer convex functions (see Property 4 in Appendix A), which establishes a direct relationship between local and global maximisers for $\overline{\mathcal{J}}$, having strong practical implications.

Property 2. [43] Let $\bar{Y}^{\text {opt }}$ be a $\Gamma$-local maximiser of $\overline{\mathcal{J}}$, i.e.

$$
\overline{\mathcal{J}}\left(\bar{Y}^{\mathrm{opt}}\right)=\max _{\bar{Y} \in \mathscr{B}\left(\bar{Y}^{\mathrm{opt}}, r\right)} \overline{\mathcal{J}}(\bar{Y}),
$$

with $r$ as in (46). Then, $\bar{Y}^{\text {opt }}$ is a global maximiser of $\overline{\mathcal{J}}$.

Property 2 (or, analogously, Property 4 in Appendix A) acts as the analogue of the global optimality property of concave functions (i.e. every local solution is a global solution). In other words, if $\bar{Y}^{\text {opt }}$ is a maximiser for $\mathscr{B}\left(\bar{Y}^{\text {opt }}, r\right)$, a subset of $\mathbb{R}^{1 \times(\nu+\iota)}$, then it is automatically a global maximiser of $\overline{\mathcal{J}}$. This not only gives explicit conditions for global energymaximisation within our nonlinear moment-based approach (in contrast to available nonlinear WEC optimal control techniques), but also considerably reduces the 'search' space when numerically solving (40), enhancing the efficiency behind the proposed moment-based strategy.

\section{On the inclusion of state and input constraints.}

As discussed in Section III-B, any energy-maximising optimal control strategy must take into account physical limitations, arising from both the device itself, and the actuator (PTO system) dynamics. Following the moment-based NP defined in Proposition 4, we propose a framework to incorporate the set of state and input constraints (12) to the energy-maximising unconstrained solution of Proposition 4.

To be precise, and in line with [15], [16], we map the set of constraints defined in (12) onto their respective momentdomain equivalents as

$$
\left\{\begin{array} { l } 
{ | x ( t ) | \leq X _ { \operatorname { m a x } } , } \\
{ | \dot { x } ( t ) | \leq V _ { \operatorname { m a x } } , } \\
{ | u ( t ) | \leq U _ { \operatorname { m a x } } , }
\end{array} \mapsto \quad \left\{\begin{array}{l}
\left|\bar{Y} S^{-1} \bar{\xi}(t)\right| \leq X_{\max }, \\
|\bar{Y} \bar{\xi}(t)| \leq V_{\max }, \\
\left|\bar{L}_{u} \bar{\xi}(t)\right| \leq U_{\max },
\end{array}\right.\right.
$$

and we enforce them only at a finite set of $N_{\rho}$ uniformlyspaced time instants $\mathcal{T}_{\rho}=\left\{t_{i}\right\}_{t=1}^{N_{\rho}} \subset \Xi$, i.e. using a collocation approach. To that end, we define the matrices $\bar{\Lambda} \in \mathbb{R}^{(\nu+\iota) \times N_{\rho}}$ and $\bar{\Delta} \in \mathbb{R}^{(\nu+\iota) \times 2 N_{\rho}}$ as

$$
\bar{\Lambda}=\left[\begin{array}{lll}
\bar{\xi}\left(t_{1}\right) & \ldots & \bar{\xi}\left(t_{N_{\rho}}\right)
\end{array}\right], \quad \bar{\Delta}=\left[\begin{array}{ll}
\bar{\Lambda} & -\bar{\Lambda}
\end{array}\right] .
$$

Finally, we can formulate a moment-based energy-maximising constrained optimal control solution for WECs, subject to nonlinear dynamics, as follows.

Corollary 3 (Nonlinear moment-based constrained NP). Suppose Assumptions 4, 5 and 6 hold, and let $\bar{\xi}(0)=\varepsilon_{\nu+\iota}$. Then, for $\iota$ sufficiently large, the solution of the approximated energymaximising OCP, posed in Problem 2, subject to the set of state and input constraints (48), can be computed as $\bar{u}^{\text {opt }}=\bar{L}_{u}^{o p t} \xi$, where

$$
\bar{L}_{u}^{\mathrm{opt}}=-\left(\bar{Y}^{\mathrm{opt}}+\operatorname{vec}\left\{F_{n l}\left(g\left(\bar{Y}^{\mathrm{opt}}\right)\right)\right\}^{\top} \Phi_{\Omega}^{\top}-\bar{L}_{e}\right) \bar{\Phi}^{-1^{\top}},
$$

and the matrix $\bar{Y}^{\mathrm{opt}}$ is the solution of the inequalityconstrained finite-dimensional nonlinear program

$$
\bar{Y}^{\text {opt }}=\arg \max _{\bar{Y}^{\top} \in \mathbb{R}^{\nu+\iota}} \overline{\mathcal{J}}_{Q P}(\bar{Y})+\overline{\mathcal{J}}_{n l}(\bar{Y}),
$$


subject to:

$$
\left\{\begin{array}{l}
\bar{Y} \mathcal{A}_{x} \leq \mathcal{B}_{x}, \\
\bar{Y} \mathcal{A}_{\dot{x}} \leq \mathcal{B}_{\dot{x}} \\
\bar{Y} \mathcal{A}_{u}+\mathcal{N}_{u}(\bar{Y}) \leq \mathcal{B}_{u},
\end{array}\right.
$$

where

$$
\begin{array}{rlrl}
\mathcal{A}_{x} & =\bar{S}^{-1} \bar{\Delta}, & \mathcal{B}_{x} & =X_{\max } \mathbf{1}_{1 \times 2 N_{\rho}}, \\
\mathcal{A}_{\dot{x}} & =\bar{\Delta}, & \mathcal{B}_{\dot{x}} & =V_{\max } \mathbf{1}_{1 \times 2 N_{\rho}}, \\
\mathcal{A}_{u} & =-\bar{\Phi}^{-1^{\top} \bar{\Delta},} & \mathcal{B}_{u} & =U_{\max } \mathbf{1}_{1 \times 2 N_{\rho}}+\bar{L}_{e} \mathcal{A}_{u}, \\
\mathcal{N}_{u}(\bar{Y}) & =-\operatorname{vec}\left\{F_{n l}(g(\bar{Y}))\right\}^{\top} \Phi_{\Omega}^{\top} \mathcal{A}_{u} .
\end{array}
$$

Proof. Note that under the set of assumptions considered in this corollary, equations (50) and (51) follow directly from Proposition 4. With respect to the incorporation of the set of state and input constraints defined in (48), let us first consider the constraint associated with the control input, and note that

$$
\left|\bar{L}_{u} S^{-1} \bar{\xi}(t)\right| \leq U_{\max } \Rightarrow-U_{\max } \leq \bar{L}_{u} \bar{\xi}(t) \leq U_{\max } .
$$

Equation (54), enforced at the set of collocation instants $\mathcal{T}_{\rho}$, can be straightforwardly written in terms of the matrix $\bar{\Delta}$ defined in (49), i.e.

$$
\bar{L}_{u} \bar{\Delta} \leq U_{\max } \mathbf{1}_{1 \times 2 N_{\rho}} .
$$

The left hand side of equation (55) can be expanded using the result of Corollary 2 as

$$
\bar{L}_{u} \bar{\Delta}=\bar{Y} \mathcal{A}_{u}-\bar{L}_{e} \mathcal{A}_{u}+\operatorname{vec}\left\{F_{n l}(g(\bar{Y}))\right\}^{\top} \Phi_{\Omega}^{\top} \mathcal{A}_{u},
$$

from where both the matrix $\mathcal{B}_{u}$ and the nonlinear mapping $\mathcal{N}_{u}$ follow directly. Finally, the claim of this corollary follows by writing the set of constraints associated with displacement and velocity, defined in (48), as in equation (55), i.e. in terms of the matrix $\bar{\Delta}$.

Remark 20. The set of inequality constraints associated with the displacement and the velocity are linear in $\bar{Y}$. This is not the case for the control input-related constraint, which can be decomposed as the sum of a linear and a nonlinear mapping $\mathcal{N}_{u}: \mathbb{R}^{1 \times(\nu+\iota)} \rightarrow \mathbb{R}^{1 \times 2 N_{\rho}}$.

Remark 21. Other types of constraints different from those considered in equation (48), e.g. limits on maximum rated power [44], unidirectional power flow [11], or specific nonlinear constraints on the control force arising from selecting a specific PTO system [45], can be incorporated into the presented moment-based approach by following an analogous procedure to that described in this section. In other words, these constraints can be included by a suitable mapping onto their respective moment-domain equivalents, followed by a (time) collocation approach.

\section{CASE STUdy: A CORPower-LiKe DeVICE.}

To demonstrate the performance of the nonlinear momentbased controller proposed in Section IV-B, we consider a fullscale state-of-the-art CorPower-like wave energy device oscillating in heave (translational motion). The actual CorPower device is a wave energy system currently under development by the Swedish company CorPower Ocean ${ }^{11}$, with the aim of making a mass and volume-efficient solution using bottomreferenced heaving buoys. The development of such a WEC builds heavily on research results and earlier experience, and the interested reader is referred to, for instance, [47], [48]. Note that this type of device is often considered as a case study, due to its intrinsic geometrical complexity (see, for example, [49]). Figure 2 presents a schematic illustration of the CorPower-like WEC, along with its corresponding hydrodynamic characterisation, i.e. the frequency-response $K(j \omega)$ associated with the impulse response mapping $k$. Note that $K(j \omega)$ has been computed using the boundary element method solver NEMOH [50]. The dimensions of this device are based on the experimental study performed in [48].
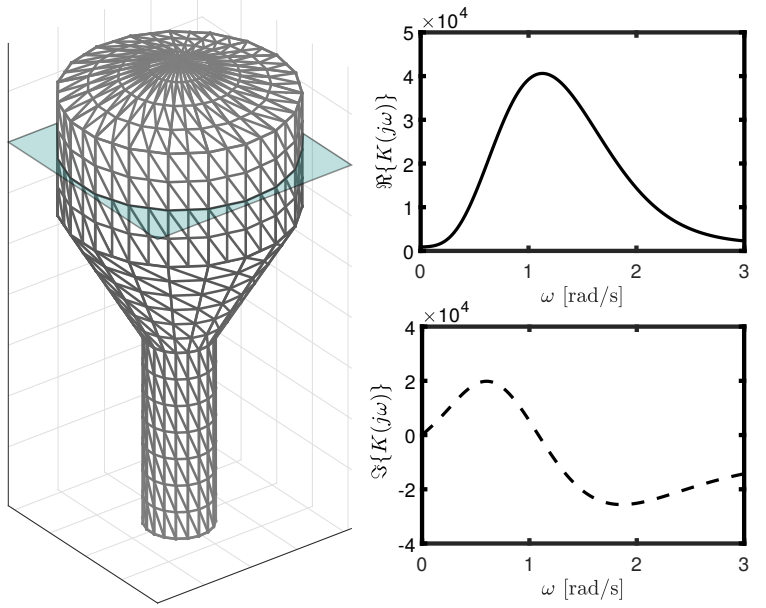

Fig. 2. Schematic of the CorPower-like device, along with the frequencyresponse of the radiation impulse response mapping $k$. The still water level is indicated using a blue-coloured (shaded) plane.

In the remainder of this section, we consider waves generated stochastically from a JONSWAP spectrum [51], with fixed significant wave height $H_{s}$ of 2 [m], varying peak period $T_{p} \in[5,12][\mathrm{s}]$, peak shape parameter $\gamma=3.3$, and a total time-length (fundamental period) of $T=120$ [s]. The corresponding spectral density functions are illustrated, for reference, in Figure 3. Since the waves are generated from sets of random amplitudes [30], it is found that a mean of $\approx 40$ simulations (per sea state) is necessary to obtain statistically consistent performance results for the nonlinear moment-based controller presented in this study, particularly those discussed in Figure 5.

\section{A. Characterisation of nonlinear hydrodynamic effects.}

In this section we characterise the nonlinear effects associated with the CorPower-like device, i.e. the mapping $\mathcal{F}^{\text {nl }}$ in (11), considered for this case study. In particular, we take into account two main hydrodynamic forces: viscous effects $\mathcal{F}_{v}$, and the presence of a nonlinear restoring force $\mathcal{F}_{r}^{n l}$, so that $\mathcal{F}^{n l}=\mathcal{F}_{v}+\mathcal{F}_{r}^{n l}$. We give explicit motivation behind the consideration of these nonlinear effects in the upcoming paragraphs.

\footnotetext{
${ }^{11}$ See [46] for up-to-date information on the CorPower device
} 


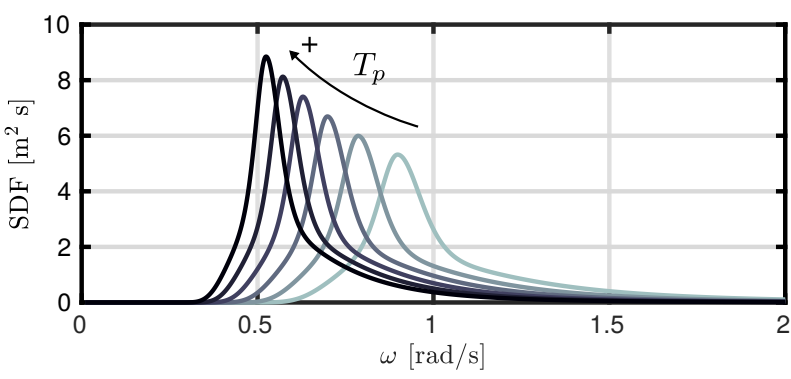

Fig. 3. Spectral density functions (SDF) for different JONSWAP spectra with fixed wave height of $H_{s}=2[\mathrm{~m}]$ and varying peak period $T_{p} \in[5,12]$ (the direction of increasing $T_{p}$ is indicated by the arrow). The peak shape parameter is fixed to $\gamma=3.3$.

Viscous effects, arising from vortex shedding and turbulence, are particularly present in heaving point absorber devices [52], such as the CorPower-like WEC considered. One common way of including viscous drag effects in the equation of motion is by means of a Morison-like term [53], i.e. we define

$$
\mathcal{F}_{v}(\dot{x})=-\beta_{v}|\dot{x}| \dot{x},
$$

where $\beta_{v}=\frac{1}{2} \rho \gamma_{d} D, \gamma_{d} \in \mathbb{R}^{+}$is the so-called drag coefficient, and $D$ is the characteristic area of the device (as defined in Section III-A). The drag coefficient is set to $\gamma_{d}=0.35$, based on the analysis performed in [7] for the device of Figure 2. Figure 4 (right axis, dashed line) illustrates the output of the mapping $\mathcal{F}_{v}$.

The mapping $\mathcal{F}_{r}^{n l}$, characterising nonlinear restoring effects, is computed based on the experimental results presented in [48] for this full-scale CorPower device. In particular, inspired by [48], we define

$$
\mathcal{F}_{r}^{n l}(x)=\beta_{r_{1}} x^{2}+\beta_{r_{2}} x^{3},
$$

where the coefficients $\left\{\beta_{r_{1}}, \beta_{r_{2}}\right\} \subset \mathbb{R}$ are determined based on a least-squares fit, using the experimental results of [48] as target set, giving a final result of $\beta_{r_{1}}=-1.55 \times 10^{4}\left[\mathrm{~kg} / \mathrm{ms}^{2}\right]$ and $\beta_{r_{2}}=0.82 \times 10^{4}\left[\mathrm{~kg} / \mathrm{m}^{2} \mathrm{~s}^{2}\right]$. The output of the nonlinear restoring force mapping $\mathcal{F}_{r}^{n l}$ is presented in Figure 4 (left axis, solid line).

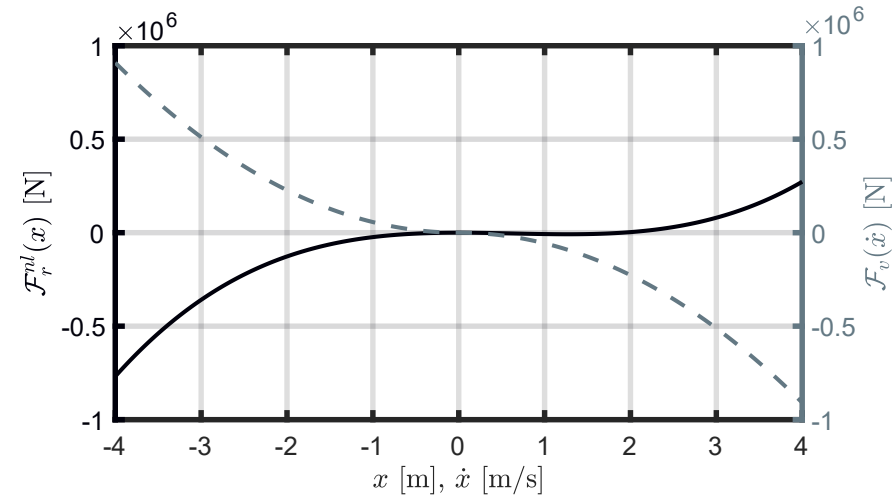

Fig. 4. Nonlinear hydrodynamic effects considered in this study: hydrostatic force (displacement-dependent, left axis), and viscous force (velocitydependent, right axis).

Note that both nonlinear effects, as described in equations (57) and (58), fulfill Assumption 5. To show that Assumption 7 holds, recall that the energy-maximising optimal control law is such that the state-variables $\varphi_{1}=x$ and $\varphi_{2}=\dot{x}$ have maximum allowed values $X_{\max }$ and $V_{\max }$, respectively (see equation (48)). Then, the following inequality, involving the nonlinear mapping $\mathcal{F}^{n l}=\mathcal{F}_{v}+\mathcal{F}_{r}^{n l}$,

$$
\begin{aligned}
\left|\mathcal{F}^{n l}(\varphi)\right| & =\beta_{v}\left|\varphi_{2}\right|^{2}+\beta_{1}\left|\varphi_{1}\right|^{2}+\beta_{2}\left|\varphi_{2}\right|^{3} \\
& \leq \beta_{v} V_{\max }+\left(\beta_{1}+\beta_{2} X_{\max }\right) X_{\max }^{2}=\tilde{\alpha}_{n l},
\end{aligned}
$$

holds for all $t \in \Xi$. Using equation (59), and considering well-known (Euclidean) norm properties, it is straightforward to show that

$$
\left\|\operatorname{vec}\left\{F_{n l}(g(\bar{Y}))\right\}\right\|_{2} \leq(\nu+\iota) \tilde{\alpha}_{n l}^{2} .
$$

Recalling, from the set of moment-domain constraints (48), that $|\bar{Y} \bar{\xi}(t)| \leq V_{\max }$ for all $t \in \Xi$, and, if $\bar{\xi}(0)=\varepsilon_{\nu+\iota}$, then $\|\bar{\xi}\|_{2}^{2}=\sum_{i=1}^{\nu+\iota}\left\langle\bar{\xi}_{i}, \bar{\xi}_{i}\right\rangle=T(\nu+\iota) / 2$, we can directly obtain the following estimate for $\alpha_{n l}$ in equation (45):

$$
\begin{aligned}
\left|\overline{\mathcal{J}}_{n l}(\bar{Y})\right| & \leq \frac{1}{2 T}\left(V_{\max }\left\|\bar{\Phi}^{-1} \Phi_{\Omega}\right\|_{F} \tilde{\alpha}_{n l}\right)^{2} \\
& =\alpha_{n l}<+\infty,
\end{aligned}
$$

and, hence, the moment-based energy-maximising OCP always admits a global maximiser under the effect of the nonlinear dynamics defined in $\mathcal{F}^{n l}$ (see Proposition 5).

\section{B. Results and discussion}

Based on the CorPower-like device of Figure 11, subject to the nonlinear effects described in Section V-A, we now present and discuss the results of applying the nonlinear momentbased energy-maximising control strategy developed throughout Section IV, under the effect of irregular (polychromatic) wave excitation forces.

We begin by setting the maximum allowed displacement and velocity values as $X_{\max }=2[\mathrm{~m}]$ and $V_{\max }=2[\mathrm{~m} / \mathrm{s}]$. The wave excitation force $\mathcal{F}_{e}$ is computed using $\nu=60$ components in (18), whilst the order of the extended signal generator (20) is set to $\nu+\iota=100$. Note that the latter effectively defines the 'size' of the optimisation variable, i.e. $\bar{Y}^{\top} \in \mathbb{R}^{\nu+\iota}$. With respect to the collocation instants used to enforce the constraints, as in Section IV-C, we have selected ten uniformly distributed collocation points per second of simulation, i.e. $N_{\rho}=1200$. The constrained moment-based optimal control problem stated in Corollary 3 can be solved using a variety of state-of-the-art numerical routines, belonging to the families of both local and global optimisation methods.

In this study, we opt for a local IPM, where we take explicit advantage of the strict outer convexity of the energy-related objective function when mapped to the moment-domain: we use Property 2 to numerically ensure that the (potentially local) solution computed with interior-point methods is, effectively, a global energy-maximiser. In particular, we propose the following simple algorithm, written in pseudo-code.

\section{1: init algorithm}

2: Set initial guess $\bar{Y}_{0}^{\text {opt }}=\bar{Y}_{l}^{\text {opt }}$;

3: global $=0$;

4: while global $\neq 1$ do

5: $\quad \bar{Y}^{\text {opt }} \leftarrow$ Solve the OCP (51) using IPM with $\bar{Y}_{0}^{\text {opt }}$; 
6: $\quad$ Generate a random set $\mathcal{P}=\left\{\bar{Y}_{i}\right\}_{i=1}^{P}$ with $P>0$ (integer) elements, such that $\mathcal{P} \subset \mathscr{B}\left(\bar{Y}^{\text {opt }}, r\right)$ and where $\bar{Y}_{i}$ is such that (52) holds for all $i \in \mathbb{N}_{P}$;

7: $\quad$ if $\overline{\mathcal{J}}\left(\bar{Y}^{\text {opt }}\right) \leq \overline{\mathcal{J}}\left(\bar{Y}_{i}\right)$ for all $\bar{Y}_{i} \in \mathcal{P}$ then

8: $\quad$ global $=1$;

9: $\quad$ else if $\exists \underline{Y}_{i} \in \mathcal{P}$ such that $\overline{\mathcal{J}}\left(\bar{Y}_{i}\right) \leq \overline{\mathcal{J}}\left(\bar{Y}^{\text {opt }}\right)$ then

10: $\quad \bar{Y}_{0}^{\mathrm{opt}}=\bar{Y}_{i}$;

11: end if

12: end while

13: $\bar{L}_{u}^{\mathrm{opt}}=-\left(\bar{Y}^{\mathrm{opt}}+\operatorname{vec}\left\{F_{n l}\left(g\left(\bar{Y}^{\mathrm{opt}}\right)\right)\right\}^{\top} \Phi_{\Omega}^{\top}-\bar{L}_{e}\right) \bar{\Phi}^{-1^{\top}}$;

14: $\bar{u}^{\mathrm{opt}}=\bar{L}_{u}^{\mathrm{opt}} \bar{\xi}$

15: end algorithm.

Starting from the linear solution $\bar{Y}_{l}^{\text {opt }}$ of the concave QP problem of Corollary 3, i.e. with $F_{n l}(g(\bar{Y}))=0$, this heuristic attempts to compute a local solution using IPMs, and simply uses function evaluation at a finite set of $P$ random points contained in the set $\mathscr{B}\left(\bar{Y}^{\text {opt }}, r\right)$, to (approximately) determine whether the solution corresponds to a global maximiser using the result expressed by Property 2 . If we can find an element $\bar{Y}_{i}$, contained in the set $\mathcal{P}$, such that $\overline{\mathcal{J}}\left(\bar{Y}_{i}\right) \leq \overline{\mathcal{J}}\left(\bar{Y}^{\text {opt }}\right)$, then the algorithm is re-started, but now updating the initial guess for the IPM to $\bar{Y}_{i}$.

Remark 22. For the nonlinear mapping associated with the CorPower-like device defined in Section V-A, the heuristic discussed above provides a global solution virtually always after a single iteration (with the cardinality of the set $\mathcal{P}$ chosen as $P=50$ ). In particular, comparisons have been carried out against global optimisation routines based on genetic algorithms (GA), to determine whether the solution obtained with the proposed heuristic effectively coincides with that computed by GA. The interior-point method utilised to solve (51) is based on [54]. Note that a range of other numerical optimisation methods can be equally applied to solve (51).

Remark 23. The moment-based controller normalised runtime, i.e. the ratio between the time required to compute the energy-maximising optimal control input for the duration of the simulation, and the length of the simulation itself, is always less than a second for the totality of the preceding simulations, which is consistent with the typical sampling time of a fullscale WEC [15], hence achieving real-time performance. We note that the real-time application of the proposed technique can be performed in a receding-horizon fashion, by directly following the moment-based methodology described in [32, Section IV] (see also Remark 4).

We now present performance results for the proposed nonlinear moment-based controller, in terms of energy absorption, under both displacement and velocity constraints. Figure 5 explicitly shows the value of $\overline{\mathcal{J}}$ (black circles), for sea states with $H_{s}=2[\mathrm{~m}]$ and $T_{p} \in[5,12]$, where the displacement and velocity of the CorPower-like device are constrained to $X_{\max }=2[\mathrm{~m}]$ and $X_{\max }=2[\mathrm{~m} / \mathrm{s}]$, respectively. In addition, Figure 5 demonstrates the performance of the linear momentbased controller (grey diamonds), i.e. solving the OCP in Corollary 3 assuming that $\overline{\mathcal{J}}_{n l}$ is zero, applied to the nonlinear system described by (14). It can be readily appreciated that the performance of the proposed nonlinear approach outperforms its linear counterpart, for the totality of the sea-states analysed in this study, with differences of up to $\approx 45 \%$ in total power absorption. We note that, though not penalised in the results of Figure 5 (to offer a best-case scenario for the linear controller), the solution based on linear assumptions can often violate the physical limitations imposed as state constraints, as a direct consequence of ignoring nonlinear effects in the computation of such an energy-maximising control law. This is illustrated and discussed in the following paragraph, where we fully expose the capabilities of the nonlinear moment-based control strategy presented in this study.

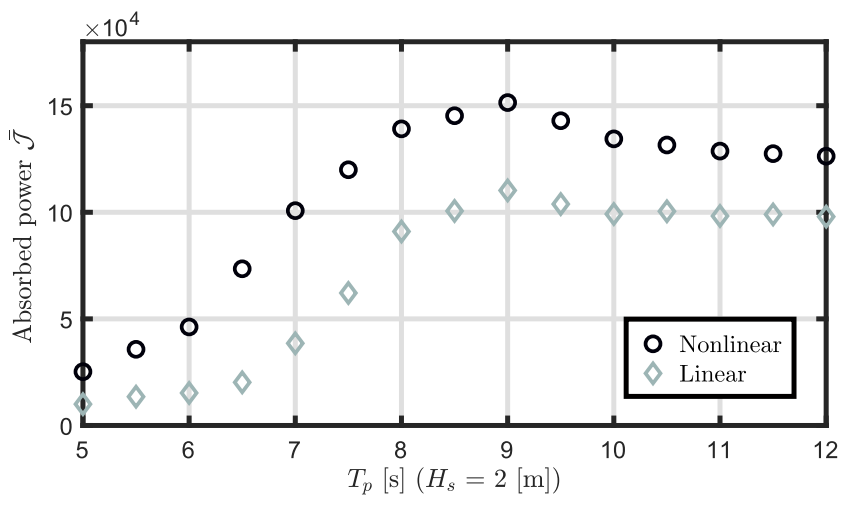

Fig. 5. Constrained (displacement and velocity) power absorption for the nonlinear moment-based energy maximising controller proposed in this paper (black circles), and its linear counterpart (grey diamonds).

Figure 6 presents time histories of displacement (a), velocity (b) and control input (c), for a specific example of sea-state realisation with $T_{p}=10[\mathrm{~s}]$, and where we have also included a maximum control (PTO) force constraint $U_{\max }=1 \times 10^{6}$ $[\mathrm{N}]$. Some key features associated with the presented momentbased strategy can be directly appreciated from Figure 6, as discussed in the following. To begin with, the state and input limits, under the action of the nonlinear moment-based control strategy (solid black), are being consistently respected throughout the complete simulation, hence illustrating the capability of the approach to maximise energy absorption for WECs subject to nonlinear hydrodynamic effects, whilst respecting the physical limitations of both device and actuator (PTO). This is clearly not the case for the solution based on linear assumptions (dotted black), where a consistent violation can be appreciated, for both displacement and velocity (state constraints). Though not significant (in magnitude) for this particular sea state, this violation happens consistently in time and can potentially damage device components.

Finally, we note that, as can be appreciated in Figure 6 (b), the velocity of the device under optimal control conditions, for both linear and nonlinear moment-based controllers, remains "in-phase"12 with the (scaled) wave excitation force (dashdotted blue), agreeing with well-known theoretical results for unconstrained energy-maximisation of WECs [24].

\footnotetext{
${ }^{12}$ We use the term 'in-phase' to indicate that the peaks (local maxima and minima) of both signals are aligned in time.
} 
(a)

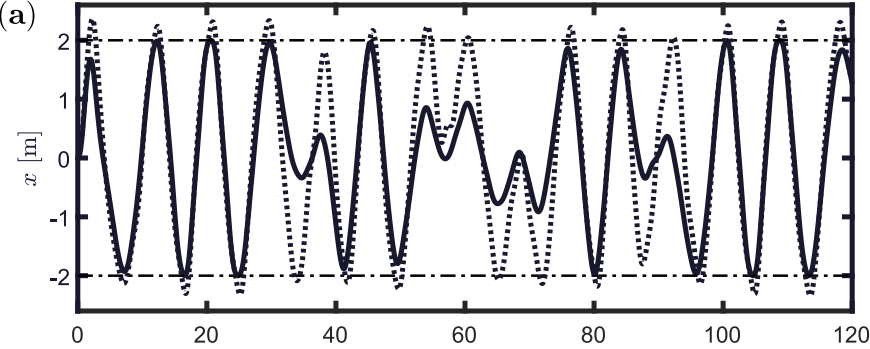

(b)
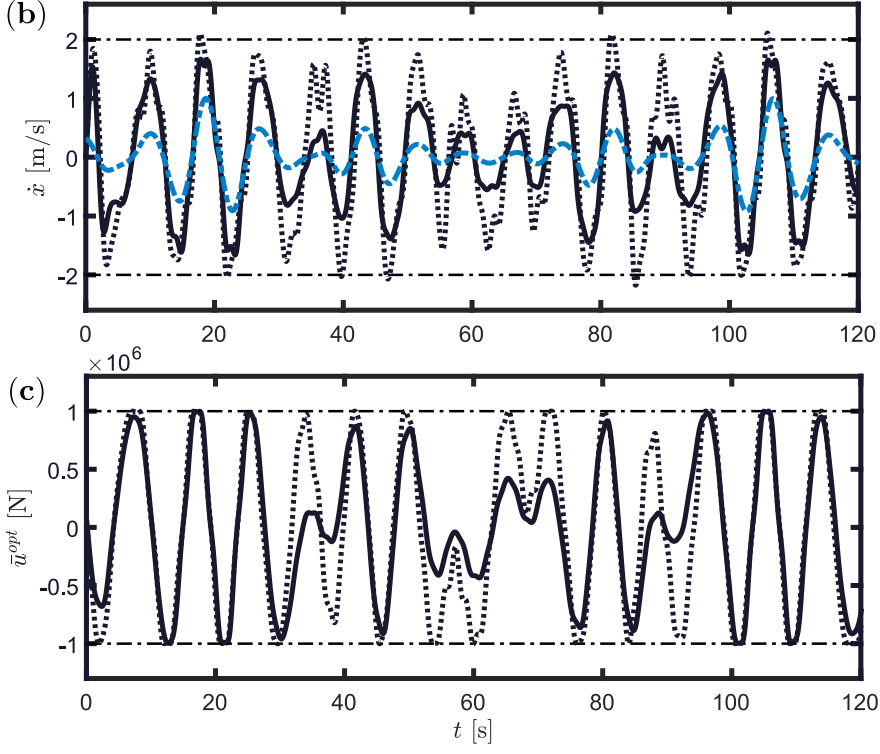

Fig. 6. Motion and control results for polychromatic wave excitation with $H_{s}=2[\mathrm{~m}]$ and $T_{p}=10[\mathrm{~s}]$, for both linear (dotted black) and nonlinear (solid black) moment-based controllers. (a) shows displacement, (b) velocity and (scaled) wave excitation force input (dash-dotted blue), whilst (c) presents the corresponding control inputs, used to elicit the motion results. The dashdotted horizontal lines represent constraint values.

\section{CONCLUSIONS}

This study introduces a nonlinear moment-based energymaximising control framework for wave energy converters, subject to both state and input constraints. The use of nonlinear moments, in conjunction with an appropriate approximation method (based on the family of weighted residual methods), allows the objective function, associated with the energymaximising OCP, to be mapped to a finite-dimensional nonlinear program, which can be solved efficiently by state-of-theart numerical solvers. We guarantee the existence of a globally optimal solution within the presented framework, under mild assumptions. In addition, we give explicit conditions that relate local and global optima, which are effectively exploited in the numerical implementation. The performance of this method is illustrated through a case study, where a CorPower-like device is considered, subject to nonlinear hydrostatic restoring force and viscous forces. We show that physical limitations are consistently respected within this nonlinear moment-based framework, maximising absorbed energy while effectively minimising the risk of component damage. Comparisons are presented with its linear counterpart, i.e. a moment-based controller without explicitly considering hydrodynamic nonlinearities when computing the control law, consistently showing improved performance for the totality of the sea states analysed, with up to $\approx 45 \%$ of increase in energy absorption. Finally, future work will compare the presented nonlinear moment-based approach with established techniques in the WEC control field, both in terms of computational speed, and energy-maximising performance.

\section{APPENDIX A \\ OUTER $\Gamma$-CONVEXITY}

To keep the paper self-contained, we recall some facts from [43]. These are particularly useful for the results derived in Section IV-B. In particular, we begin by recalling the definition of outer $\Gamma$-convexity, originally proposed in [19], [43].

Definition 5 (Outer $\Gamma$-convexity [19], [43]). Let $\Gamma \subset \mathbb{R}^{n}$ and $g: \mathbb{R}^{n} \rightarrow \mathbb{R}$. The function $g$ is said to be outer $\Gamma$-convex on a given nonempty convex set $D \subset \mathbb{R}^{n}$ if, for all $\left\{x_{0}, x_{1}\right\} \subset D$ satisfying $x_{0}-x_{1} \notin \Gamma$, there is a closed subset $Z \subset[0,1]$ containing $\{0,1\}$ such that

$$
\left[x_{0}, x_{1}\right] \subset\left\{(1-\zeta) x_{0}+\zeta x_{1} \mid \zeta \in Z\right\}+\frac{1}{2} \Gamma,
$$

and

$$
g\left((1-\zeta) x_{0}+\zeta x_{1}\right) \leq(1-\zeta) g\left(x_{0}\right)+\zeta g\left(x_{1}\right),
$$

$\forall \zeta \in Z \backslash\{0,1\}$. If the inequality (63) holds strictly, then $g$ is said to be strictly outer $\Gamma$-convex.

Definition 5 has shown to be useful in deriving specific properties of boundedly perturbed strictly convex quadratic functions [43]. In particular, let $A \in \mathbb{R}^{n \times n}$ be a symmetric positive definite matrix, $b \in \mathbb{R}^{n}$, and

$$
f(x):=x^{\top} A x+b^{\top} x,
$$

with $x \in \mathbb{R}^{n}$. For a given set $D \subset \mathbb{R}^{n}$, equation (64) gives origin to the convex quadratic program, termed problem $(P)$ :

$$
\min _{x \in D} f(x) .
$$

Consider now the modified problem $(\tilde{P})$ :

$$
\min _{x \in D} \tilde{f}(x):=f(x)+p(x),
$$

where $p: \mathbb{R}^{n} \rightarrow \mathbb{R}$ is lower semi-continuous. For convenience, we call $p$ the perturbation, $\tilde{f}$ the perturbed function and $(\tilde{P})$ the perturbed problem. We now recall the following fundamental result from [43].

Theorem 2. [43, Theorem 2.2]. Suppose the perturbation $p$ is bounded by a parameter s, i.e. $\sup _{x \in D}|p(x)| \leq s<+\infty$. Then the perturbed function $\tilde{f}=f+p$ is strictly outer $\Gamma$ convex on $D$ for $\Gamma=\mathscr{B}(0, r)$, with $r=\sqrt{2 s / \min \lambda(A)}$.

The result of Theorem 2 shows that a bounded perturbation $p$ does not completely destroy the strict convexity of the quadratic function $f(x)=x^{\top} A x+b^{\top} x$, but the perturbed function $\tilde{f}=f+p$ is still strictly outer $\Gamma$-convex for some suitable set $\Gamma \subset \mathbb{R}^{n}$. This has two fundamental implications for the perturbed problem $(\tilde{P})$, which we recall below as properties. Note that we explicitly state the source of each of these properties, by pointing to specific results of [43]. In 
what follows, we shall assume the perturbation $p$ is bounded by a parameter, so that Theorem 2 can be used.

Property 3. [43, Lemma 4.3]. Let $x_{\mathrm{QP}}^{*}$ be the global minimiser of problem $(P)$ and suppose the set $\mathscr{B}\left(x_{\mathrm{QP}}^{*}, r\right) \cap D$ is closed. Then problem $(\tilde{P})$ always admits a global minimiser.

Property 4. [43, Theorem 3.1]. Let $x^{*}$ be a local $\Gamma$-minimiser of $\tilde{f}$, i.e.

$$
\tilde{f}\left(x^{*}\right)=\min _{x \in \mathscr{B}\left(x^{*}, r\right) \cap D} \tilde{f}(x) .
$$

Then $x^{*}$ is a global minimiser of $\tilde{f}$, i.e.

$$
\tilde{f}\left(x^{*}\right)=\min _{x \in D} \tilde{f}(x) .
$$

\section{REFERENCES}

[1] U. A. Korde and J. Ringwood, Hydrodynamic control of wave energy devices. Cambridge University Press, 2016.

[2] J. V. Ringwood, G. Bacelli, and F. Fusco, "Energy-maximizing control of wave-energy converters: The development of control system technology to optimize their operation," IEEE Control Systems, vol. 34, no. 5, pp. 30-55, 2014.

[3] N. Faedo, S. Olaya, and J. V. Ringwood, "Optimal control, MPC and MPC-like algorithms for wave energy systems: An overview," IFAC Journal of Systems and Control, vol. 1, pp. 37-56, 2017.

[4] E. Anderlini, D. Forehand, E. Bannon, and M. Abusara, "Reactive control of a wave energy converter using artificial neural networks," International journal of marine energy, vol. 19, pp. 207-220, 2017.

[5] D. Valério, M. J. Mendes, P. Beirão, and J. S. da Costa, "Identification and control of the aws using neural network models," Applied Ocean Research, vol. 30, no. 3, pp. 178-188, 2008.

[6] M. Penalba, G. Giorgi, and J. V. Ringwood, "Mathematical modelling of wave energy converters: a review of nonlinear approaches," Renewable and Sustainable Energy Reviews, vol. 78, pp. 1188-1207, 2017.

[7] G. Giorgi and J. V. Ringwood, "A compact 6-dof nonlinear wave energy device model for power assessment and control investigations," IEEE Transactions on Sustainable Energy, vol. 10, no. 1, pp. 119-126, 2018.

[8] M. Richter, M. E. Magaña, O. Sawodny, and T. K. Brekken, "Nonlinear model predictive control of a point absorber wave energy converter," IEEE Transactions on Sustainable Energy, vol. 4, no. 1, pp. 118-126, 2013.

[9] N. Tom and R. W. Yeung, "Nonlinear model predictive control applied to a generic ocean-wave energy extractor," Journal of Offshore Mechanics and Arctic Engineering, vol. 136, no. 4, p. 041901, 2014.

[10] K. U. Amann, M. E. Magaña, and O. Sawodny, "Model predictive control of a nonlinear 2-body point absorber wave energy converter with estimated state feedback," IEEE Transactions on Sustainable Energy, vol. 6, no. 2, pp. 336-345, 2014.

[11] G. Bacelli, R. Genest, and J. V. Ringwood, "Nonlinear control of flaptype wave energy converter with a non-ideal power take-off system," Annual Reviews in Control, vol. 40, pp. 116-126, 2015.

[12] C. Auger, A. P. L. Merigaud, and J. V. Ringwood, "Receding-horizon pseudo-spectral control of wave energy converters using periodic basis functions," IEEE Transactions on Sustainable Energy, 2018.

[13] G. Li, "Nonlinear model predictive control of a wave energy converter based on differential flatness parameterisation," International Journal of Control, pp. 1-10, 2015.

[14] I. M. Ross and M. Karpenko, "A review of pseudospectral optimal control: From theory to flight," Annual Reviews in Control, vol. 36, no. 2, pp. 182-197, 2012.

[15] N. Faedo, G. Scarciotti, A. Astolfi, and J. V. Ringwood, "Energymaximising control of wave energy converters using a moment-domain representation," Control Engineering Practice, vol. 81, pp. 85 - 96, 2018.

[16] _ - "Moment-based constrained optimal control of an array of wave energy converters," in 2019 American Control Conference (ACC), Philadelphia, 2019.

[17] A. Astolfi, "Model reduction by moment matching for linear and nonlinear systems," IEEE Transactions on Automatic Control, vol. 55 , no. 10 , pp. 2321-2336, 2010.

[18] D. P. Bertsekas, "Nonlinear programming," Journal of the Operational Research Society, vol. 48, no. 3, pp. 334-334, 1997.
[19] H. X. Phu, "Outer $\gamma$-convexity in vector spaces," Numerical Functional Analysis and Optimization, vol. 29, no. 7-8, pp. 835-854, 2008.

[20] J. Brewer, "Kronecker products and matrix calculus in system theory," IEEE Transactions on circuits and systems, vol. 25, no. 9, pp. 772-781, 1978.

[21] G. Scarciotti and A. Astolfi, "Nonlinear model reduction by moment matching," Foundations and Trends in Systems and Control, vol. 4, no. 3-4, pp. 224-409, 2017.

[22] A. Isidori, Nonlinear control systems. Springer Science \& Business Media, 2013.

[23] A. Padoan, G. Scarciotti, and A. Astolfi, "A geometric characterization of the persistence of excitation condition for the solutions of autonomous systems," IEEE Transactions on Automatic Control, vol. 62, no. 11, pp. 5666-5677, 2017.

[24] J. Falnes, Ocean waves and oscillating systems: linear interactions including wave-energy extraction. Cambridge university press, 2002.

[25] P. Tona, H.-N. Nguyen, G. Sabiron, and Y. Creff, "An efficiencyaware model predictive control strategy for a heaving buoy wave energy converter," in Proceedings of the 11th European Wave and Tidal Energy Conference (EWTEC), Nantes, France, 2015, p. $01443855 f$.

[26] W. Cummins, "The impulse response function and ship motions," DTIC Document, Tech. Rep., 1962.

[27] A.-M. Wazwaz, "Volterra integro-differential equations," in Linear and Nonlinear Integral Equations. Springer, 2011, pp. 175-212.

[28] G. Bacelli and J. V. Ringwood, "A geometric tool for the analysis of position and force constraints in wave energy converters," Ocean Engineering, vol. 65, pp. 10-18, 2013.

[29] N. Faedo, "Optimal control and model reduction for wave energy systems: A moment-based approach." Ph.D. dissertation, Department of Electronic Engineering, Maynooth University, 2020.

[30] A. Mérigaud and J. V. Ringwood, "Free-surface time-series generation for wave energy applications," IEEE Journal of Oceanic Engineering, 2017.

[31] Y. Peña-Sanchez, M. Garcia-Abril, F. Paparella, and J. V. Ringwood, "Estimation and forecasting of excitation force for arrays of wave energy devices," IEEE Transactions on Sustainable Energy, 2018.

[32] N. Faedo, Y. Peña-Sanchez, and J. V. Ringwood, "Receding-horizon energy-maximising optimal control of wave energy systems based on moments," IEEE Transactions on Sustainable Energy (early access available), 2020.

[33] J. Scruggs, S. Lattanzio, A. Taflanidis, and I. Cassidy, "Optimal causal control of a wave energy converter in a random sea," Applied Ocean Research, vol. 42, pp. 1-15, 2013.

[34] J.-P. Aubin, Applied functional analysis. John Wiley \& Sons, 2011, vol. 47.

[35] J. J. Benedetto, Harmonic analysis and applications. CRC Press, 1996, vol. 23.

[36] A. Isidori and A. Astolfi, "Disturbance attenuation and $H_{\infty}$-control via measurement feedback in nonlinear systems," IEEE transactions on automatic control, vol. 37, no. 9, pp. 1283-1293, 1992.

[37] G. Scarciotti and A. Astolfi, "Data-driven model reduction by moment matching for linear and nonlinear systems," Automatica, vol. 79, pp. 340-351, 2017.

[38] B. Finlayson and L. Scriven, "The method of weighted residualsa review," Appl. Mech. Rev, vol. 19, no. 9, pp. 735-748, 1966.

[39] J. P. Boyd, Chebyshev and Fourier spectral methods. Courier Corporation, 2001

[40] M. Urabe, "Galerkin's procedure for nonlinear periodic systems," Archive for Rational Mechanics and Analysis, vol. 20, no. 2, pp. 120$152,1965$.

[41] G. Scarciotti and A. Astolfi, "Moment-based discontinuous phasor transform and its application to the steady-state analysis of inverters and wireless power transfer systems," IEEE Transactions on Power Electronics, vol. 31, no. 12, pp. 8448-8460, 2016.

[42] A. Forsgren, P. E. Gill, and M. H. Wright, "Interior methods for nonlinear optimization," SIAM review, vol. 44, no. 4, pp. 525-597, 2002.

[43] H. Phu and V. Pho, "Some properties of boundedly perturbed strictly convex quadratic functions," Optimization, vol. 61, no. 1, pp. 67-88, 2012.

[44] A. C. O'Sullivan and G. Lightbody, "Co-design of a wave energy converter using constrained predictive control," Renewable Energy, vol. 102, pp. 142-156, 2017.

[45] G. P. R. Papini, G. Moretti, R. Vertechy, and M. Fontana, "Control of an oscillating water column wave energy converter based on dielectric elastomer generator," Nonlinear Dynamics, vol. 92, no. 2, pp. 181-202, 2018. 
[46] "CorPower Ocean," http://www.corpowerocean.com/, accessed: 31-10 2019.

[47] M. Lopes, J. Hals, R. Gomes, T. Moan, L. Gato, and A. Falcão, "Experimental and numerical investigation of non-predictive phasecontrol strategies for a point-absorbing wave energy converter," Ocean Engineering, vol. 36, no. 5, pp. 386-402, 2009.

[48] J. Hals-Todalshaug, G. S. Ásgeirsson, E. Hjálmarsson, J. Maillet, P. Möller, P. Pires, M. Guérinel, and M. Lopes, "Tank testing of an inherently phase-controlled wave energy converter," International Journal of Marine Energy, vol. 15, pp. 68-84, 2016.

[49] G. Giorgi and J. V. Ringwood, "Analytical representation of nonlinear Froude-Krylov forces for 3-DoF point absorbing wave energy devices," Ocean Engineering, vol. 164, pp. 749-759, 2018.

[50] A. Babarit and G. Delhommeau, "Theoretical and numerical aspects of the open source BEM solver NEMOH," in 11th European Wave and Tidal Energy Conference, Nantes, 2015.

[51] K. Hasselmann, "Measurements of wind wave growth and swell decay during the Joint North Sea Wave Project (JONSWAP)," Dtsch. Hydrogr. Z., vol. 8, p. 95, 1973.

[52] G. Giorgi and J. V. Ringwood, "Nonlinear Froude-Krylov and viscous drag representations for wave energy converters in the computation/fidelity continuum," Ocean Engineering, vol. 141, pp. 164-175, 2017.

[53] J. Morison, J. Johnson, S. Schaaf et al., "The force exerted by surface waves on piles," Journal of Petroleum Technology, vol. 2, no. 05, pp. 149-154, 1950.

[54] R. A. Waltz, J. L. Morales, J. Nocedal, and D. Orban, "An interior algorithm for nonlinear optimization that combines line search and trust region steps," Mathematical programming, vol. 107, no. 3, pp. 391-408, 2006.

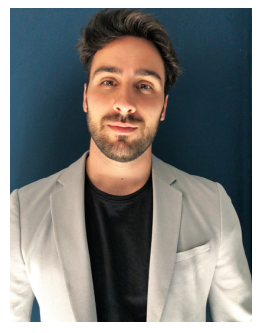

Nicolás Faedo was born in Buenos Aires, Argentina, in 1991. He received the degree in Automation and Control engineering from the National University of Quilmes, Buenos Aires, Argentina, in 2015. In 2017, he joined the Centre for Ocean Energy Research group, Maynooth University, Ireland, where he obtained a Ph.D. degree in Electronic Engineering in 2020 with a thesis on optimal control and model reduction for wave energy systems. He was a visiting researcher at the Control and Power Group, Imperial College London, in 2018. He is the recipient of the 2019 ISOPE Best Student Paper Award and Exxonmobile Prize, and finalist for the 2018 IFAC CAMS Young Author Award.

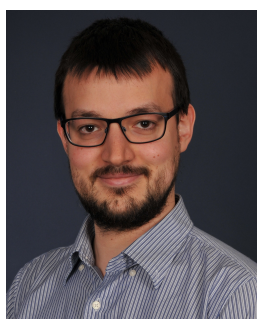

Giordano Scarciotti was born in Frascati (Rome), Italy, in 1988. He received his B.Sc. and M.Sc. degree in Automation Engineering from the University of Rome Tor Vergata, Italy, in 2010 and 2012, respectively. In 2012 he joined the Control and Power Group, Imperial College London, London, UK, where he obtained a Ph.D. degree in 2016 with a thesis on approximation, analysis and control of large-scale systems. He is currently a Lecturer (Assistant Professor) in the Control and Power Group. His current research interests are focused on analysis and control of uncertain systems (modelled by stochastic equations and setvalued mappings), model reduction and optimal control. He was a visiting scholar at New York University in 2015 and at University of California Santa Barbara in 2016. He is the recipient of an Imperial College Junior Research Fellowship (2016), of the IET Control \& Automation PhD Award (2016), the Eryl Cadwaladr Davies Prize (2017) and of an ItalyMadeMe award (2017).

$\mathrm{He}$ is a member of the IEEE CSS Conference Editorial Board, of the IFAC Technical Committee in Nonlinear Control Systems and of the IPC of the IFAC World Congress 2020. He is the Local Organisation Chair for the EUCA European Control Conference 2022.

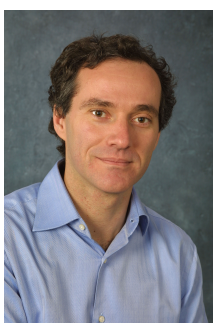

Alessandro Astolfi was born in Rome, Italy, in 1967. He graduated in electrical engineering from the University of Rome in 1991. In 1992 he joined ETH-Zurich where he obtained a M.Sc. in Information Theory in 1995 and the Ph.D. degree with Medal of Honor in 1995 with a thesis on discontinuous stabilization of nonholonomic systems. In 1996 he was awarded a Ph.D. from the University of Rome La Sapienza for his work on nonlinear robust control. Since 1996 he has been with the Electrical and Electronic Engineering Department of Imperial College London, London (UK), where he is currently Professor of Nonlinear Control Theory and Head of the Control and Power Group. From 1998 to 2003 he was also an Associate Professor at the Dept. of Electronics and Information of the Politecnico of Milano. Since 2005 he has also been a Professor at Dipartimento di Ingegneria Civile e Ingegneria Informatica, University of Rome Tor Vergata. He has been a visiting lecturer in Nonlinear Control in several universities, including ETH-Zurich (1995-1996); Terza University of Rome (1996); Rice University, Houston (1999); Kepler University, Linz (2000); SUPELEC, Paris (2001), Northeastern University (2013).

His research interests are focused on mathematical control theory and control applications, with special emphasis for the problems of discontinuous stabilization, robust and adaptive control, observer design and model reduction. $\mathrm{He}$ is the author of more than 150 journal papers, of 30 book chapters and of over 240 papers in refereed conference proceedings. He is the author (with D. Karagiannis and R. Ortega) of the monograph "Nonlinear and Adaptive Control with Applications" (Springer-Verlag).

$\mathrm{He}$ is the recipient of the IEEE CSS A. Ruberti Young Researcher Prize (2007), the IEEE RAS Googol Best New Application Paper Award (2009), the IEEE CSS George S. Axelby Outstanding Paper Award (2012), the Automatica Best Paper Award (2017). He is a "Distinguished Member" of the IEEE CSS, IEEE Fellow and IFAC Fellow. He served as Associate Editor for Automatica, Systems and Control Letters, the IEEE Trans. on Automatic Control, the International Journal of Control, the European Journal of Control and the Journal of the Franklin Institute; as Area Editor for the Int. J. of Adaptive Control and Signal Processing; as Senior Editor for the IEEE Trans. on Automatic Control; and as Editor-in-Chief for the European Journal of Control. He is currently Editor-in-Chief of the IEEE Trans. on Automatic Control. He served as Chair of the IEEE CSS Conference Editorial Board (2010- 2017) and in the IPC of several international conferences. He was/is a Member of the IEEE Fellow Committee $(2016,2019,2020)$.

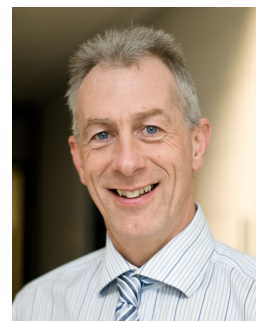

John V. Ringwood John V. Ringwood (M87-SM97) received the Diploma in Electrical Engineering from the Dublin Institute of Technology, Ireland, and the $\mathrm{Ph} . \mathrm{D}$. degree in control systems from Strathclyde University, Glasgow, U.K. He was with the School of Electronic Engineering, Dublin City University, and has held visiting positions with Massey University, Palmerston North, New Zealand, and The University of Auckland, New Zealand. He is currently Professor of Electronic Engineering and the Director of the Centre for Ocean Energy Research with Maynooth University, Ireland. He is a Chartered Engineer and a Fellow of Engineers Ireland.

His research interests focus on control systems and its applications, including renewable energy systems (and wave energy in particular), physiology and exercise physiology. He is the co-author (with Umesh Korde) of the monograph "Hydrodynamic Control of Wave Energy Devices" and was corecipient of the IEEE 2016 Control Systems Outstanding Paper award. He is currently Associate Editor for IEEE Transactions on Sustainable Energy, the Journal of Ocean Engineering and Marine Energy, and IET Renewable Power Generation. 